\title{
Post-Newtonian approximation for isolated systems calculated by matched asymptotic expansions
}

\author{
Olivier Poujade and Luc Blanchet \\ Institut d'Astrophysique de Paris (C.N.R.S.), \\ $98^{\text {bis }}$ boulevard Arago, 75014 Paris, France
}

(October 26, 2018)

\begin{abstract}
Two long-standing problems with the post-Newtonian approximation for isolated slowly-moving systems in general relativity are : (i) the appearance at high post-Newtonian orders of divergent Poisson integrals, casting a doubt on the soundness of the post-Newtonian series; (ii) the domain of validity of the approximation which is limited to the near-zone of the source, and prevents one, a priori, from incorporating the condition of no-incoming radiation, to be imposed at past null infinity. In this article, we resolve the problem (i) by iterating the post-Newtonian hierarchy of equations by means of a new (Poisson-type) integral operator that is free of divergencies, and the problem (ii) by matching the post-Newtonian near-zone field to the exterior field of the source, known from previous work as a multipolar-post-Minkowskian expansion satisfying the relevant boundary conditions at infinity. As a result, we obtain an algorithm for iterating the post-Newtonian series up to any order, and we determine the terms, present in the post-Newtonian field, that are associated with the gravitational-radiation reaction onto an isolated slowly-moving matter system.

4.25.Nx, 04.30.-w
\end{abstract}

Typeset using REVTEX 


\section{INTRODUCTION}

\section{A. Problems with the post-Newtonian expansion}

The post-Newtonian approximation, or expansion when the speed of light $c \rightarrow+\infty$, has been formalized in the early days of general relativity by Einstein [1], Droste [2], and DeSitter [3]. Since then, it has provided us with our best insights about the problems of motion and gravitational radiation, two of general relativity's most important issues. Concerning the problem of motion, we quote the dynamics of $N$ separated bodies at the first post-Newtonian (1PN, or $\left.1 / c^{2}\right)$ order : works of Einstein, Infeld and Hoffmann [4] and other authors [5], and the dynamics of extended fluid systems up to the 2.5PN level of gravitational radiation reaction : works of Chandrasekhar and collaborators [8 10 and followers [11]19. In the case of two compact objects, we know the 2.5PN equations of motion of the binary pulsar 20 23], and the 3PN equations of motion of inspiralling compact binaries 24 28]. The specific contribution of the gravitational-radiation reaction has been obtained up to the $1.5 \mathrm{PN}$ relative order by the method of matched asymptotic expansions for extended fluids [29 33], and by means of balance equations for compact binary systems 34,35. Concerning the problem of gravitational radiation, the work has focused on the expressions of the multipole

moments of general fluid systems [36 40], and on the gravitational-wave flux emitted by inspiralling compact binaries, including the specific effects of wave tails, up to the 3.5PN order 414 45].

The "standard" post-Newtonian approximation, at the basis of most of the body of work quoted previously, is known to be plagued with some apparently inherent difficulties, which crop up at some high post-Newtonian order like 3PN. Up to the 2.5PN order the approximation can be worked out without problems, and at the $3 \mathrm{PN}$ order the problems can be solved specifically for each case at hands (see for instance Ref. [27]). However, it must be admitted that these difficulties, even appearing at higher approximations, cast doubt on the actual soundness, on the theoretical point of view, of the post-Newtonian 
expansion. What is maybe worse, they pose the practical question of the reliability of this approximation when comparing the theory's predictions with very precise experimental results. It is therefore highly desirable to assess the nature of these difficulties - are they purely technical or linked with some fundamental drawback of the approximation scheme? - and eventually to resolve them. This is especially important in view of the fact that inspiralling compact binaries, when they are detected and analyzed by gravitational-wave experiments, will necessitate a prior theoretical knowledge of the gravitational-wave signal at some very high post-Newtonian order 41 45. In this article let us distinguish (and resolve) the two basic problems faced by the post-Newtonian expansion.

The first problem is that in higher approximations some divergent Poisson-type integrals appear. Recall that the post-Newtonian expansion replaces the resolution of an hyperboliclike d'Alembertian equation by a perturbatively equivalent hierarchy of elliptic-like Poisson equations. Rapidly it is found during the post-Newtonian iteration that the right-hand-side of the Poisson equations acquires a non-compact support (it is distributed over all space), and that the standard Poisson integral diverges because of the bound of the integral at spatial infinity, i.e. $r \equiv|\mathbf{x}| \rightarrow+\infty$, with $t=$ const. For instance some of the potentials occuring at the 2PN order in Chandrasekhar's work [9] are divergent, so the corresponding metric is formally infinite 1. In fact, Kerlick [14,15] showed that the post-Newtonian computation à la Chandrasekhar [8 10], following the iteration scheme of Anderson and DeCanio [11], can be made well-defined up to the $2.5 \mathrm{PN}$ order, by keeping some derivatives inside some crucial integrals to make them finite 12,13 . However, the latter remedy does not solve the problem at the next 3PN order, which has been found to involve some inexorably divergent Poisson integrals [14, 115].

These divergencies come from the fact that the post-Newtonian expansion is actually a

\footnotetext{
${ }^{1}$ Nevertheless, these divergencies were not a problem when considering the equations of motion because the gradients of these potentials, which parametrize the equations, were finite.
} 
singular perturbation, in the sense that the coefficients of the successive powers of $1 / c$ are not uniformly valid in space, since they typically blow up at spatial infinity like some positive powers of $r$. For instance, Rendall [46] has shown that the post-Newtonian expansion cannot be "asymptotically flat" starting at the 2PN or 3PN level, depending on the adopted coordinate system. The result is that the Poisson integrals are in general badly-behaving at infinity. Physically this can be understood by the fact that the post-Newtonian approximation is valid only in the near zone of the source (see below) while the Poisson integral extends over the whole three-dimensional space, including the regions far from the source where the approximation breaks down. Therefore, trying to solve the post-Newtonian equations by means of the standard Poisson integral does not a priori make sense. This does not mean that there are no solution to the problem, but simply that the Poisson integral does not constitute the correct solution of the Poisson equation in the context of post-Newtonian expansions. So the difficulty is purely of a technical nature, and will be solved once we succeed in finding the appropriate solution to the Poisson equation f]. A solution to the problem of divergencies has been proposed by Futamase and Schutz [47] and Futamase 448. Their approach is alternative to the one we shall follow below. It is based on an initialvalue formalism, which avoids the appearance of divergencies because of the finiteness of the integration region.

The second problem has to do with the near-zone limitation of the approximation. Indeed

\footnotetext{
${ }^{2}$ The problem is somewhat similar to what happens in Newtonian cosmology. Here we have to solve the Poisson equation $\Delta U=-4 \pi G \rho$, where the density $\rho$ of the cosmological fluid is constant all over space : $\rho=\rho(t)$. Clearly the Poisson integral of a constant density does not make sense, as it diverges at the bound at infinity like the integral $\int r d r$. This nonsensical result has occasionally been referred to as the "paradox of Seeliger". However the problem is solved once we realize that the Poisson integral does not constitute the appropriate solution of the Poisson equation in the context of Newtonian cosmology. A well-defined solution is simply given by $U=-\frac{2}{3} \pi G \rho r^{2}$.
} 
the post-Newtonian expansion assumes that all retardations $r / c$ are small, so it can be viewed as a formal near-zone expansion when $r \rightarrow 0$, which is valid only in the region surrounding the source that is of small extent with respect to the typical wavelength of the emitted radiation : $r \ll \lambda$ (if we locate the origin of the coordinates $r=0$ inside the source). Therefore, the fact that the coefficients of the post-Newtonian expansion blow up at spatial infinity, when $r \rightarrow+\infty$, has nothing to do with the actual behaviour of the field at infinity. The serious consequence is that it is not possible, a priori, to implement within the postNewtonian iteration the physical information that the matter system is isolated from the rest of the universe. Most importantly, the no-incoming radiation condition, imposed at past null infinity, cannot be taken into account, a priori, into the scheme. In a sense the postNewtonian approximation is not "self-supporting", because it necessitates some information taken from outside its own domain of validity.

To the lowest post-Newtonian orders one can circumvent this difficulty by considering retarded integrals that are formally expanded when $c \rightarrow+\infty$ as series of "instantaneous" Poisson-like integrals 11]. This procedure works well up to the 2.5PN level and has been shown to correctly fix the dominant radiation reaction term at the 2.5PN order [14,15]. Unfortunately such a procedure assumes fundamentally that the gravitational field, after expansion of all retardations $r / c \rightarrow 0$, depends on the state of the source at a single time, in keeping with the instantaneous character of the Newtonian interaction. However, we know that from the 4PN order the post-Newtonian field (as well as the source's dynamics) ceases to be given by a functional of the source parameters at a single time, because of the imprint of gravitational-wave tails in the near zone field, in the form of some modification, at the $1.5 \mathrm{PN}$ relative order, of the radiation reaction force [31-33. Therefore, the formal post-Newtonian expansion of retarded Green functions is no longer valid starting at the 4PN order. We face here a true difficulty, which is fundamentally linked to the nature of the post-Newtonian approximation.

The aim of the present article is to resolve the two latter problems. We shall prove that the post-Newtonian expansion can be indefinitely reiterated, while incorporating the 
correct boundary conditions satisfied by the wave field at infinity. In particular, we shall get new insights about the problem of gravitational-radiation reaction inside an isolated (post-Newtonian) system. To cure the problem of divergencies we introduce, at any postNewtonian order, a generalized solution of the Poisson equation with non-compact support source, in the form of an appropriate finite part of the usual Poisson integral : namely we regularize the bound at infinity of the Poisson integral by means of a process of analytic continuation, analogous to the one already used to regularize the retarded integrals in Refs. [36,39,40]. Our generalized solution constitutes a particular (well-defined) solution of the problem; the most general solution is the sum of that particular solution and the most general solution of the corresponding homogeneous equation, i.e. the source-free Laplace equation. The homogeneous solution should be regular all over the matter system (we are considering smooth matter distributions), and we find, after summing up the post-Newtonian series, that it can be thoroughly written with the help of some tensorial functions of time $A_{L}^{\mu \nu}(t)$, where $L=i_{1} \cdots i_{l}$ denotes a multi-index with $l$ indices 49. At this stage, considering the post-Newtonian iteration scheme alone, we cannot do more and therefore we leave the functions $A_{L}^{\mu \nu}(t)$ unspecified. We refer to them as some "radiation-reaction" functions.

The solution of the problem of the near-zone limitation of the post-Newtonian expansion resides in the matching of the near-zone field to the exterior field, a solution of the vacuum equations outside the source which has been developed in previous works [36,32] using some post-Minkowskian and multipolar expansions. In the case of post-Newtonian sources, the near zone, i.e. $r \ll \lambda$, covers entirely the source, because the source's radius itself is such that $a \ll \lambda$. Thus the near zone overlaps with the exterior zone where the multipole expansion is valid. Matching together the post-Newtonian and multipolar-post-Minkowskian solutions in this overlapping region is an application of the method of matched asymptotic expansions, and has frequently been applied in the present context, both for radiation-reaction [29 33] and wave-generation 3740 problems.

The exterior multipolar-post-Minkowskian field originally obtained in Ref. [36] depends on some "multipole-moment" functions, say $X_{L}^{\mu \nu}(t)$ [whose components are associated with 
some source multipole moments e.g. $\left.I_{L}(t), J_{L}(t), \cdots\right]$, which must be left unspecified as long as we consider only the external vacuum solution. In the work [40], we have shown that the multipole moments $X_{L}^{\mu \nu}(t)$ are entirely determined, up to any post-Newtonian order, from the requirement of matching to a post-Newtonian solution. In the present paper, we shall further show that the radiation-reaction functions $A_{L}^{\mu \nu}(t)$, parametrizing the post-Newtonian solution, are also uniquely fixed, up to any post-Newtonian order, by the matching. In particular, we shall find that the latter functions include correctly the contribution of wave tails, arising at the 4PN order, as determined in Refs. 31 33]. We shall also recover by a different method the result of Ref. [40] concerning the multipole moments $X_{L}^{\mu \nu}(t)$.

A comment is in order regarding the possibility of determining the near-zone field by matched asymptotic expansions up to any post-Newtonian order. Indeed the method presupposes the existence of the exterior near-zone for which $a<r \ll \lambda$. Now if a given physical system, whose dynamics is described by Newton's theory, emits gravitational radiation at some Newtonian fundamental wave length $\lambda_{\mathrm{N}}$, we expect that when taking into account the post-Newtonian corrections up to the post-Newtonian order $n$, it will have a radiation spectrum composed of harmonics between $\sim 2 \lambda_{\mathrm{N}} / n$ and $\sim 2 \lambda_{\mathrm{N}}$. Indeed this is the case of the radiation from a binary system moving on a circular orbit, for which we have $\frac{2}{n+2} \lambda_{\mathrm{N}} \leq$ $\lambda_{n \mathrm{PN}} \leq 2 \lambda_{\mathrm{N}}$. Therefore, if $n$ is large enough, say $n \gtrsim 2 \lambda_{\mathrm{N}} / a$, we expect that there will be some part of the radiation whose frequency is too high for the exterior near zone to exist. What we want to say is that the formulas we shall obtain for the post-Newtonian field of a source "up to any order" are indeed physically valid, strictly speaking, only up to some finite post-Newtonian order $\sim 2 \lambda_{\mathrm{N}} / a$, where $a$ is the size of the source; but that, if we consider a source which is less relativistic, for instance which is obtained by "slowing down" our source so that its Newtonian wave length gets twice its original value (say), the same post-Newtonian formulas can then be used for the new source up to approximately twice the previous post-Newtonian order.

The plan of this paper is as follows. In Section $\mathbb{1}$ we recall the construction in Ref. [36] of the multipole expansion of the external field, and we obtain thanks to a result of 
Ref. [32 the near-zone expansion of that external field ready for subsequent matching. In Section [II] we implement the post-Newtonian iteration of the inner field inside the matter source, and we find the far-zone (multipolar) expansion of that post-Newtonian solution, also ready for matching. In Section $\mathbb{\mathbb { }} \nabla$ we show that the matching works up to any postNewtonian order, and permits the determination of all the unknowns, in both the external and inner fields. Finally in Section $\bigvee$ we check that our post-Newtonian solution satisfies the harmonic-coordinate condition as a consequence of the equations of motion of the source. The technical proofs are relegated to Appendices A, B and Q .

\section{B. Notation for the Einstein field equations}

For the problem at hands let us introduce an asymptotically Minkowskian coordinate system for which the basic gravitational-wave amplitude, $h^{\mu \nu}=\sqrt{-g} g^{\mu \nu}-\eta^{\mu \nu}$, is divergenceless, i.e. satisfies the de Donder or harmonic gauge condition $\partial_{\mu} h^{\mu \nu}=0$. Here, $g^{\mu \nu}$

denotes the contravariant metric (satisfying $\left.g^{\mu \rho} g_{\rho \nu}=\delta_{\nu}^{\mu}\right), g$ is the determinant of the covariant metric, $g=\operatorname{det}\left(g_{\mu \nu}\right)$, and $\eta^{\mu \nu}$ represents an auxiliary Minkowskian metric with signature +2 . With these definitions the Einstein field equations can be recast into the d'Alembertian equation

$$
\square h^{\mu \nu}=\frac{16 \pi G}{c^{4}} \tau^{\mu \nu},
$$

where $\square=\eta^{\mu \nu} \partial_{\mu} \partial_{\nu}=-\frac{1}{c^{2}} \partial^{2} / \partial t^{2}+\Delta$ is the (flat-spacetime) d'Alembertian operator. The source term, $\tau^{\mu \nu}$, can rightly be interpreted as the "effective" stress-energy pseudo-tensor of the matter and gravitational fields in harmonic coordinates. It is conserved in the usual sense, and that is equivalent to the condition of harmonic coordinates :

$$
\partial_{\mu} h^{\mu \nu}=0 \Longleftrightarrow \partial_{\mu} \tau^{\mu \nu}=0
$$


The pseudo-tensor $\tau^{\mu \nu}$ is made of the contribution of the matter fields, described by a stressenergy tensor $T^{\mu \nu}$, and the one due to the gravitational field, given by the gravitational source term $\Lambda^{\mu \nu} ;$ thus,

$$
\tau^{\mu \nu}=|g| T^{\mu \nu}+\frac{c^{4}}{16 \pi G} \Lambda^{\mu \nu}
$$

The conservation property (1.2) is equivalent to the conservation, in the covariant sense, of the matter tensor : $\nabla_{\mu} T^{\mu \nu}=0$. The exact expression of $\Lambda^{\mu \nu}$, taking into account all the non-linearities of the Einstein field equations, reads

$$
\begin{aligned}
\Lambda^{\mu \nu}= & -h^{\rho \sigma} \partial_{\rho \sigma}^{2} h^{\mu \nu}+\partial_{\rho} h^{\mu \sigma} \partial_{\sigma} h^{\nu \rho}+\frac{1}{2} g^{\mu \nu} g_{\rho \sigma} \partial_{\lambda} h^{\rho \tau} \partial_{\tau} h^{\sigma \lambda} \\
& -g^{\mu \rho} g_{\sigma \tau} \partial_{\lambda} h^{\nu \tau} \partial_{\rho} h^{\sigma \lambda}-g^{\nu \rho} g_{\sigma \tau} \partial_{\lambda} h^{\mu \tau} \partial_{\rho} h^{\sigma \lambda}+g_{\rho \sigma} g^{\lambda \tau} \partial_{\lambda} h^{\mu \rho} \partial_{\tau} h^{\nu \sigma} \\
& +\frac{1}{8}\left(2 g^{\mu \rho} g^{\nu \sigma}-g^{\mu \nu} g^{\rho \sigma}\right)\left(2 g_{\lambda \tau} g_{\epsilon \pi}-g_{\tau \epsilon} g_{\lambda \pi}\right) \partial_{\rho} h^{\lambda \pi} \partial_{\sigma} h^{\tau \epsilon} .
\end{aligned}
$$

It is clear from this expression that $\Lambda^{\mu \nu}$ is made of terms which are at least quadratic in the gravitational-field strength $h^{\mu \nu}$ and its first and second space-time derivatives.

In this article, we look for the solutions of the field equations (1.1)-(1.4) under the following hypotheses. First, we assume that the matter tensor $T^{\mu \nu}$ has a spatially compact support, i.e. can be enclosed into some time-like world tube, say $r \leq a$, where $r=|\mathbf{x}|$ is the harmonic-coordinate radial distance. Second, we assume that the matter distribution inside the source is smooth : i.e. $T^{\mu \nu}(\mathbf{x}, t) \in C^{\infty}\left(\mathbb{R}^{4}\right)$. We have in mind a smooth hydrodynamical "fluid" system, without any singularities nor shocks (a priori), that is described by some Eulerian-type equations including high relativistic (post-Newtonian) corrections. In particular, we exclude from the start any sources containing black holes. Notice, however, that it makes sense to apply the formulas derived a priori only for smooth matter distributions to systems containing compact objects (including black holes), described by some sort of point-particle singularities; see e.g. Refs. 41-45. Finally, in order to select the physically sensible solution of the field equations, we choose some boundary conditions at infinity corresponding to the famous no-incoming radiation condition. In this paper, we shall rely on 
a specific construction of the metric outside the domain of the source $(r>a)$, that was achieved in Ref. [36] under the assumption that the gravitational field has been independent of time (stationary) in some remote past, in the sense that $t \leq-\mathcal{T} \Longrightarrow \frac{\partial}{\partial t}\left[h^{\mu \nu}(\mathbf{x}, t)\right]=0$. This condition is a mean to impose, by brute force, the no-incoming radiation condition $\mathrm{p}$.

\section{EXTERIOR FIELD}

\section{A. Multipolar expansion of the non-linear vacuum field}

In this section we review some material from Ref. [36] concerning the construction of vacuum metrics by means of mixed multipolar and post-Minkowskian (MPM) expansions. The so-called MPM metrics aim at describing the gravitational field in the region exterior to a general isolated system. In fact they are mathematically defined in the open domain $\mathbb{R}_{*}^{3} \times \mathbb{R}$, i.e. $\mathbb{R}^{4}$ deprived from the spatial origin $r \equiv|\mathbf{x}|=0$, but of course do not agree physically with the real solution when $0<r<a$, since they are vacuum solutions. For our present purpose the point is that the most general physically admissible solution of the vacuum field equations has been obtained in Ref. [36] by a specific construction of the post-Minkowskian solution, say

$$
h_{\mathrm{ext}}^{\mu \nu}=\sum_{m=1}^{+\infty} G^{m} h_{(m)}^{\mu \nu},
$$

whose coefficients are in the form of multipolar series, or equivalently decompositions in symmetric-trace-free (STF) products of unit vectors $\hat{n}_{L}$, that are equivalent to the usual decomposition in spherical harmonics 49 :

\footnotetext{
${ }^{3}$ However the condition of stationarity in the past, though much weaker than the actual noincoming radiation condition, does not seem to entail any physical restriction on the applicability of the formalism, even in the case of sources which have always been radiating.
} 


$$
\forall m \geq 1, \quad h_{(m)}^{\mu \nu}(\mathbf{x}, t)=\sum_{l=0}^{+\infty} \hat{n}_{L}(\theta, \phi) h_{(m) L}^{\mu \nu}(r, t) .
$$

The $h_{(m) L}^{\mu \nu}$ 's are certain functions of the radial coordinate $r$ and of time $t$. Inserting the MPM expansion (2.1)-(2.2) into the vacuum field equations (1.1)-(1.2) we obtain, at any post-Minkowskian order $m$,

$$
\begin{aligned}
\square h_{(m)}^{\mu \nu} & =\Lambda_{(m)}^{\mu \nu}\left[h_{(1)}, \cdots, h_{(m-1)}\right], \\
\partial_{\mu} h_{(m)}^{\mu \nu} & =0,
\end{aligned}
$$

where $\Lambda_{(m)}^{\mu \nu}$ denotes the $m$-th post-Minkowskian piece of the gravitational source term defined by Eq. (1.4), i.e. in which we have inserted the previous post-Minkowskian iterations up to the previous order $m-1$ [with the convention that $\left.\Lambda_{(1)}^{\mu \nu}=0\right]$. Because Eq. (1.4) is at least quadratic in non-linearities, it is clear that only the preceding iterations, $\leq m-1$, are necessary at any post-Minkowskian order $m$.

Now the solution that was obtained in Ref. 36] has two main characteristics. The first one is related to its particular near-zone structure, which will play a fundamental role in the present article. Namely, it was proved that each one of the multipolar-post-Minkowskian coefficients $h_{(m)}^{\mu \nu}$ in Eq. (2.1) - that we recall are only defined when $r>0$-, admits a singular near-zone expansion, i.e. when $r \rightarrow 0$, owning the following structure :

$$
\forall N \in \mathbb{N}, \quad h_{(m)}^{\mu \nu}(\mathbf{x}, t)=\sum_{l, a, p} \hat{n}_{L} r^{a}(\ln r)^{p} F_{(m) L, a, p}^{\mu \nu}(t)+R_{(m) N}^{\mu \nu}(\mathbf{x}, t)
$$

where the multipolar order $l \in \mathbb{N}$, where the powers of $r$ are such that $a \in \mathbb{Z}$ with $a_{\min } \leq a \leq$ $N$ (with $a_{\text {min }}$ a negative integer), and where the powers of $\ln r$ are $p \in \mathbb{N}$ with $p \leq m-1$. The maximal divergence when $r \rightarrow 0$ occurs for $a_{\text {min }}$, which depends on the post-Minkowskian order $m$, and satisfies $a_{\min }(m) \rightarrow-\infty$ when $m \rightarrow+\infty$. Similarly the maximal power of the logarithms, $p_{\max }(m)=m-1$, tends to infinity with $m$. The functions $F_{(m) L, a, p}^{\mu \nu}(t)$ are smooth functions of time, $F_{(m) L, a, p}^{\mu \nu} \in C^{\infty}(\mathbb{R})$, which are to be computed by means of 
the algorithm proposed in Ref. [36], and appear as complicated non-linear functionals of some more elementary functions parametrizing the linearized $(m=1)$ approximation. The remainder term in Eq. (2.5) is such that

$$
R_{(m) N}^{\mu \nu}(\mathbf{x}, t)=\mathcal{O}\left(r^{N}\right) \quad \text { when } r \rightarrow 0 \text { and } t=\text { const } .
$$

The Landau $\mathcal{O}$-symbol takes its usual meaning. This remainder admits also some specific differentiability properties (refer to [36] for the details). The gravitational source term $\Lambda_{(m)}^{\mu \nu}$ admits exactly the same near-zone structure as in Eq. (2.5) with the exception that $p_{\max }=m-2$ in this case (that is, the maximal power of the logarithms increases by one unit when going from the source to the solution).

The second important characteristic of the MPM solution concerns the constructive formula which defines it. We find that each one of the post-Minkowskian coefficients $h_{(m)}^{\mu \nu}$ is explicitly constructed by means of the following [36] :

$$
h_{(m)}^{\mu \nu}=\underset{B=0}{\mathrm{FP}} \square_{\text {Ret }}^{-1}\left[\widetilde{r}^{B} \Lambda_{(m)}^{\mu \nu}\right]+\sum_{l=0}^{+\infty} \hat{\partial}_{L}\left\{\frac{1}{r} X_{(m) L}^{\mu \nu}\left(t-\frac{r}{c}\right)\right\} .
$$

The first term involves a special type of generalized inverse d'Alembertian operator, built on the standard retarded integral,

$$
\square_{\text {Ret }}^{-1}\left[\widetilde{r}^{B} \Lambda_{(m)}^{\mu \nu}\right](\mathbf{x}, t) \equiv-\frac{1}{4 \pi} \int_{\mathbb{R}^{3}} \frac{d^{3} \mathbf{y}}{|\mathbf{x}-\mathbf{y}|}|\widetilde{\mathbf{y}}|^{B} \Lambda_{(m)}^{\mu \nu}(\mathbf{y}, t-|\mathbf{x}-\mathbf{y}| / c),
$$

which extends over the whole three-dimensional space, but inside which a regularization factor has been "artificially" introduced, namely

$$
\widetilde{r}^{B} \equiv\left(\frac{r}{r_{0}}\right)^{B}
$$

where $B$ denotes a complex number, $B \in \mathbb{C}$, and $r_{0}$ represents an arbitrary constant length scale. The indication $\underset{B=0}{\mathrm{FP}}$ stands for the finite part at $B=0$, and means that one should 
first compute the Laurent expansion when $B \rightarrow 0$ of (the analytic continuation of) the $B$-dependent integral (2.8), and, second, pick up the finite part at $B=0$ in that expansion, i.e. the coefficient of the zero-th power of $B$. The main property of this generalized retarded operator, that we shall from now on abbreviate as

$$
\widetilde{\square_{\text {Ret }}^{-1}}\left[\Lambda_{(m)}^{\mu \nu}\right] \equiv \underset{B=0}{\mathrm{FP}} \square_{\text {Ret }}^{-1}\left[\widetilde{r}^{B} \Lambda_{(m)}^{\mu \nu}\right]
$$

is that, for source terms $\Lambda_{(m)}^{\mu \nu}$ admitting a near-zone structure of the type (2.5),

$$
\square\left[\widetilde{\square_{\operatorname{Ret}}^{-1}} \Lambda_{(m)}^{\mu \nu}\right]=\Lambda_{(m)}^{\mu \nu}
$$

Because the second term in Eq. (2.7) is a retarded solution of the source-free wave equation, we see therefore that $h_{(m)}^{\mu \nu}$ represents indeed a solution of the wave equation we had to solve : $\square h_{(m)}^{\mu \nu}=\Lambda_{(m)}^{\mu \nu}$. However this is not sufficient because we have also to solve the harmoniccoordinate condition (1.2). We shall refer to [36 for the definition of an algorithm which permits to compute, simply from the algebraic and differential structure of the vacuum field equations, the necessary form of the second term in Eq. (2.7), in such a way that the harmonic-coordinate condition be satisfied : $\partial_{\mu} h_{(m)}^{\mu \nu}=0$. In fact we shall not need, in the following, to be more precise about the latter term; simply we keep it into the form of a general retarded solution of the source-free wave equation, parametrized by some tensorial functions $X_{(m) L}^{\mu \nu}(t)$. We assume that these functions are STF with respect to the multi-index $L$ : i.e. $X_{(m) L}^{\mu \nu} \equiv \hat{X}_{(m) L}^{\mu \nu}$, so the multi-derivative $\hat{\partial}_{L}$ in Eq. 2.7) is a STF one (see Ref. 49 for the notation). The latter construction represents the most general physical solution of the field equations outside the source [36].

Let us now proceed with the formal re-summation of the post-Minkowskian series. That is, once the results (2.5)-(2.7) have been established for any order $m$, we sum them from $m=1$ up to infinity. In this way we obtain some formulas which are valid formally for the complete post-Minkowskian series, and, presumably, could hold true in a more rigorous 
context of exact solutions. After summation we shall "forget" about the post-Minkowskian expansion, and consider that the exterior field $h_{\mathrm{ext}}^{\mu \nu}$ represents merely the multipole decomposition of the actual field $h^{\mu \nu}$ outside the compact support of the source (nevertheless, it is wise to keep in mind that the solution came from a formal post-Minkowskian summation). We denote the multipole decomposition by means of the calligraphic letter $\mathcal{M}$. Therefore, our definition is that the multipole expansion $\mathcal{M}\left(h^{\mu \nu}\right)$ of the field outside the isolated source is nothing but the external solution constructed previously by means of the MPM method, and re-summed over the post-Minkowskian index $m$ :

$$
\mathcal{M}\left(h^{\mu \nu}\right) \equiv h_{\mathrm{ext}}^{\mu \nu}
$$

This definition is quite legitimate (and rather obvious) because we know that the MPM metric constitutes the most general solution for the exterior field. Thus, $\mathcal{M}\left(h^{\mu \nu}\right)$ is a solution of the vacuum field equations, now considered outside the physical domain of the source, $r>a$ (while $h_{\mathrm{ext}}^{\mu \nu}$ had been constructed for any $r>0$ ). In that domain we have evidently the numerical equality

$$
\mathcal{M}\left(h^{\mu \nu}\right)=h^{\mu \nu} \quad(\text { when } r>a)
$$

After summation of Eqs. (2.5)-(2.6) over $m$, we get the near-zone structure

$$
\forall N \in \mathbb{N}, \quad \mathcal{M}\left(h^{\mu \nu}\right)=\sum \hat{n}_{L} r^{a}(\ln r)^{p} F_{L, a, p}^{\mu \nu}(t)+\mathcal{O}\left(r^{N}\right)
$$

in which the functions $F_{L, a, p}^{\mu \nu}(t)=\sum_{m=1}^{+\infty} G^{m} F_{(m) L, a, p}^{\mu \nu}(t)$, and where $a \leq N$ and $p \geq 0$. Notice that there is no lower bound for $a$ because $a_{\min }(m) \rightarrow-\infty$ when $m \rightarrow+\infty$; similarly there is no upper bound for $p$. Secondly, coming to the constructive formula (2.7) we obtain

$$
\mathcal{M}\left(h^{\mu \nu}\right)=\widetilde{\square_{\text {Ret }}^{-1}}\left[\mathcal{M}\left(\Lambda^{\mu \nu}\right)\right]+\sum_{l=0}^{+\infty} \hat{\partial}_{L}\left\{\frac{1}{r} X_{L}^{\mu \nu}\left(t-\frac{r}{c}\right)\right\}
$$


where $X_{L}^{\mu \nu}(t)=\sum_{m=1}^{+\infty} G^{m} X_{(m) L}^{\mu \nu}(t)$. In the following we shall regard the STF functions $X_{L}^{\mu \nu}(t)$ as the "multipole moments" of the source, because they describe the physics of the source as seen from the exterior. We do not need to be more precise at this point. Let us simply comment that by imposing the harmonic-gauge condition (1.2) we find that there are only six components of these functions which are independent, and this yields the definition of six independent STF source multipole moments $I_{L}(t), J_{L}(t), \cdots$ (see Ref.

40 for the precise definition). Furthermore, the multipole-moment functions $X_{L}^{\mu \nu}(t)$ have already been calculated in terms of the stress-energy tensor of a post-Newtonian source in Ref. [40]. However we prefer to leave these functions undetermined because we shall recover their expressions by means of a somewhat different method, and the agreement we shall find with the result of Ref. [40] will constitute a crucial check of our computation.

\section{B. Near-zone expansion of the multipole decomposition}

In anticipation of the matching we consider next the infinite near-zone re-expansion, when $r \rightarrow 0$, of the multipole expansion $\mathcal{M}\left(h^{\mu \nu}\right)$ determined in Eq. (2.15). We have already obtained the general structure of that expansion, given by Eq. (2.14). Let us denote with the help of some overline the infinite near-zone expansion (without remainder), whose structure is therefore given by

$$
\overline{\mathcal{M}\left(h^{\mu \nu}\right)}=\sum \hat{n}_{L} r^{a}(\ln r)^{p} F_{L, a, p}^{\mu \nu}(t)
$$

where $a \in \mathbb{Z}$ and $p \in \mathbb{N}$ (and, of course, the multipolar index $l \in \mathbb{N}$ ). We must be careful at distinguishing the fully-fledged multipole decomposition $\mathcal{M}\left(h^{\mu \nu}\right)$, which is defined as soon as $r>0$ and numerically agrees with the exact solution wherever $r>a$ (in particular when $r \rightarrow+\infty)$, from its formal near-zone re-expansion $\overline{\mathcal{M}\left(h^{\mu \nu}\right)}$. Later we shall indicate the post-Newtonian expansion by means of the same overline notation. Indeed the near-zone expansion is really an expansion when $r / \lambda \rightarrow 0$, which is equivalent to an expansion when 
$c \rightarrow+\infty$, since the wavelength of waves is $\lambda=c P$ (with $P=$ typical period of the internal motion). From the result (2.15) we can write

$$
\overline{\mathcal{M}\left(h^{\mu \nu}\right)}=\overline{\widetilde{\square_{\text {Ret }}^{-1}}\left[\mathcal{M}\left(\Lambda^{\mu \nu}\right)\right]}+\sum_{l=0}^{+\infty} \hat{\partial}_{L}\left\{\frac{\overline{X_{L}^{\mu \nu}(t-r / c)}}{r}\right\} .
$$

The overline in the second term means that one should expand the retardations $t-r / c$ when $r / c \rightarrow 0$. More explicitly we have

$$
\hat{\partial}_{L}\left\{\frac{\overline{X_{L}^{\mu \nu}(t-r / c)}}{r}\right\}=\sum_{j=0}^{+\infty} \frac{(-)^{j}}{c^{j} j !} \hat{\partial}_{L}\left(r^{j-1}\right) X_{L}^{\mu \nu}(t)
$$

where the superscript $(j)$ indicates $j$ successive time-derivations. The main problem is how to treat the first term in Eq. (2.17). What we essentially want is to know how one can "commute" the operations of taking the near-zone expansion and of applying the retarded integral. In fact, the problem has already been solved in Ref. [32], which succeeded in writing the first term in Eq. (2.17) as the sum of an "instantaneous" operator, acting on the near-zone expansion of the source, and of a particular "anti-symmetric" wave (i.e. retarded minus advanced), solution of the source-free d'Alembertian equation. The result of Ref. [32], Eq. (3.2), reads

$$
\widetilde{\widetilde{\square_{\text {Ret }}^{-1}}\left[\mathcal{M}\left(\Lambda^{\mu \nu}\right)\right]}=\widetilde{\mathcal{I}^{-1}}\left[\overline{\mathcal{M}\left(\Lambda^{\mu \nu}\right)}\right]-\frac{4 G}{c^{4}} \sum_{l=0}^{+\infty} \frac{(-)^{l}}{l !} \hat{\partial}_{L}\left\{\frac{\overline{\mathcal{R}_{L}^{\mu \nu}(t-r / c)-\mathcal{R}_{L}^{\mu \nu}(t+r / c)}}{2 r}\right\}
$$

For completeness we present in Appendix A the proof of this result - a version of it which is somewhat improved with respect to that given in Ref. 32. The first term in Eq. (2.19) involves an operator $\widetilde{\mathcal{I}^{-1}}$, acting on each of the individual terms of the formal near-zone expansion whose structure is given by Eq. (2.16), and which is essentially defined by the solution of the wave equation that is obtained by iterated use of inverse Laplace operators, and regularized by means of our B-dependent finite part procedure. Thus, 


$$
\widetilde{\mathcal{I}^{-1}}\left[\overline{\mathcal{M}\left(\Lambda^{\mu \nu}\right)}\right]=\underset{B=0}{\mathrm{FP}} \sum_{k=0}^{+\infty}\left(\frac{\partial}{c \partial t}\right)^{2 k} \Delta^{-k-1}\left[\widetilde{r}^{B} \overline{\mathcal{M}\left(\Lambda^{\mu \nu}\right)}\right]
$$

where $\Delta^{-k-1}=\left(\Delta^{-1}\right)^{k+1}$, and the action of the inverse Laplacian on the generic term of Eq. (2.16) follows from

$$
\Delta^{-1}\left[\hat{n}_{L} r^{B+a}(\ln r)^{p}\right]=\left(\frac{d}{d B}\right)^{p}\left[\frac{\hat{n}_{L} r^{B+a+2}}{(B+a+2-l)(B+a+3+l)}\right]
$$

[see also Eq. ( $\overline{\mathrm{A} 16}$ ) in Appendix $\mathrm{A}]$. The operator $\widetilde{\mathcal{I}^{-1}}$ plays the central role in the present paper. It can be regarded as (the regularization of) the formal post-Newtonian expansion, when $c \rightarrow+\infty$, of the inverse d'Alembert operator, say $\mathcal{I}^{-1}=\overline{1 / \square}=\overline{1 /\left(\Delta-\frac{1}{c^{2}} \partial_{t}^{2}\right)}$. We can refer to $\mathcal{I}^{-1}$ as the operator of the instantaneous potentials, because it acts on the time variable $t$ only through time-derivations, instead of involving a full integration like for the operator of the retarded potentials $\square_{\text {Ret }}^{-1}$. Notice that $\mathcal{I}^{-1}$ is closely related to the operator of the symmetric potentials, $\frac{1}{2}\left[\square_{\text {Ret }}^{-1}+\square_{\text {Adv }}^{-1}\right]$; see Ref. 32 for discussion and the precise relation between these operators. As for the second term in Eq. (2.19), it is made of an "anti-symmetric" wave, which represents in fact a solution of the d'Alembertian equation that is regular in a neighbourhood of the origin $r=0$. Its near-zone expansion $r / c \rightarrow 0$ is composed only of terms containing some odd powers of $1 / c$ :

$$
\begin{aligned}
\hat{\partial}_{L}\left\{\frac{\overline{\mathcal{R}_{L}^{\mu \nu}(t-r / c)-\mathcal{R}_{L}^{\mu \nu}(t+r / c)}}{2 r}\right\} & =-\sum_{i=l}^{+\infty} \frac{\hat{\partial}_{L}\left(r^{2 i}\right)}{(2 i+1) !} \frac{\stackrel{(2 i+1)}{\mathcal{R}}_{L}^{\mu \nu}(t)}{c^{2 i+1}} \\
& =-\frac{1}{(2 l+1) ! !} \sum_{k=0}^{+\infty} \widetilde{\Delta^{-k}}\left(\hat{x}_{L}\right) \frac{\mathcal{R}^{\mu \nu}(t)}{c^{2 k+2 l+1}}
\end{aligned}
$$

See also Eqs. (2.4)-(2.7) in Ref. [32] for alternative forms of the anti-symmetric wave. In the second of Eqs. (2.22) we have introduced the useful object

$$
\widetilde{\Delta^{-k}}\left(\hat{x}_{L}\right)=\frac{(2 l+1) ! !}{(2 k) ! !(2 l+2 k+1) ! !} r^{2 k} \hat{x}_{L}
$$


which represents the iterated Laplacian operator $\widetilde{\Delta^{-k}}$, regularized by means of the $\underset{B=0}{\mathrm{FP}}$ process, acting on $\hat{x}_{L}$ which denotes the STF projection of the product $x_{L} \equiv x_{i_{1}} \cdots x_{i_{l}}$ 49. [See also Eq. (C18) for an alternative expression of the same object.] From Ref. [32], or from Eq. (A11) in Appendix A, we get the expression of the functions parametrizing the anti-symmetric waves,

$$
\mathcal{R}_{L}^{\mu \nu}(t)=\underset{B=0}{\mathrm{FP}} \int d^{3} \mathbf{x}|\widetilde{\mathbf{x}}|^{B} \hat{x}_{L} \int_{1}^{+\infty} d z \gamma_{l}(z) \mathcal{M}\left(\tau^{\mu \nu}\right)(\mathbf{x}, t-z|\mathbf{x}| / c),
$$

where $|\widetilde{\mathbf{x}}|=|\mathbf{x}| / r_{0}$ [see Eq. (2.9)]. These functions depend on the whole past-history of the source [50]. The $z$-integration involves the weighting function defined by

$$
\gamma_{l}(z)=(-)^{l+1} \frac{(2 l+1) ! !}{2^{l} l !}\left(z^{2}-1\right)^{l} .
$$

This function is normalized so that $\int_{1}^{+\infty} d z \gamma_{l}(z)=1$, where the value of the integral is obtained by analytic continuation for $l \in \mathbb{C}$ (see Appendix A). As shown in Ref. [32] (see notably Section III.D there), the anti-symmetric waves in Eq. (2.19) are associated with gravitational radiation reaction effects of non-linear origin. In particular they contain the contribution of wave tails in the radiation reaction force, which appears at the $1.5 \mathrm{PN}$ order relatively to the lowest-order radiation damping, i.e. $4 \mathrm{PN}$ order in the equations of motion [31]. To summarize this subsection, we have obtained the near-zone re-expansion of the multipole expansion $\mathcal{M}\left(h^{\mu \nu}\right)$ as

$$
\begin{aligned}
\overline{\mathcal{M}\left(h^{\mu \nu}\right)} & =\widetilde{\mathcal{I}^{-1}}\left[\overline{\mathcal{M}\left(\Lambda^{\mu \nu}\right)}\right] \\
& -\frac{4 G}{c^{4}} \sum_{l=0}^{+\infty} \frac{(-)^{l}}{l !} \hat{\partial}_{L}\left\{\frac{\overline{\mathcal{R}_{L}^{\mu \nu}(t-r / c)-\mathcal{R}_{L}^{\mu \nu}(t+r / c)}}{2 r}\right\} \\
& +\sum_{l=0}^{+\infty} \hat{\partial}_{L}\left\{\frac{\overline{X_{L}^{\mu \nu}(t-r / c)}}{r}\right\} .
\end{aligned}
$$

The functions $\mathcal{R}_{L}^{\mu \nu}(t)$ are known from Eq. (2.22), but the multipole-moments $X_{L}^{\mu \nu}(t)$ have not yet been specified at this stage (though they have already been calculated in Ref. [40]). 
Therefore, we have succeeded in computing the near-zone expansion $\overline{\mathcal{M}\left(h^{\mu \nu}\right)}$ as a functional of the sole unknown constituted by the $X_{L}^{\mu \nu}$ 's; only by matching can these functions be determined.

\section{INTERIOR FIELD}

\section{A. Post-Newtonian expansion of the near-zone field}

Up to now, we have solved the Einstein field equations in the vacuum outside an isolated source $(r>a)$, without any reference to the stress-energy tensor $T^{\mu \nu}$ of the matter source. Our next task is to investigate the field equations inside and in the vicinity of the matter source, and more precisely in the so-called near-zone, or region for which $r \ll \lambda$, where $\lambda$ is the typical wavelength of the emitted waves. From now on we restrict attention to a post-Newtonian source, whose radius is $a \ll \lambda$. For post-Newtonian sources the near zone overlaps with the external region in what we shall refer to as the matching region, for which $a<r \ll \lambda$. In the matching region both the multipolar expansion of the exterior field and the post-Newtonian expansion of the inner field are legitimate.

Let us denote by means of an overline the formal (infinite) post-Newtonian expansion of the field inside the source's near-zone $: \bar{h}^{\mu \nu}$, which is of the form

$$
\bar{h}^{\mu \nu}(\mathbf{x}, t, c)=\sum_{n=2}^{+\infty} \frac{1}{c^{n}} \bar{h}_{n}^{\mu \nu}(\mathbf{x}, t, \ln c) .
$$

By definition, the $n$-th post-Newtonian coefficient $\frac{h_{n}}{n}$ is the factor of the $n$-th power of $1 / c$; however, we know from the structure of the near-zone expansion of the exterior field [see Eq. (2.16)] that the post-Newtonian expansion will involve also, besides the usual powers of $1 / c$, some logarithms of $c$ (in fact when stating this we are anticipating on the result of the matching). So the coefficients $\bar{h}_{n}^{\mu \nu}$ still depend on $c$ via the logarithm of $\ln c$, and from Eq. (2.16) we infer that they are in fact some power series in $\ln c$. The first appearance of $\ln c$ is at the $4 \mathrm{PN}$ order (i.e. corresponding to a term $\sim \ln c / c^{8}$ in the equations of motion) and is 
associated to the physical effect of wave tails [31]. In Eq. (3.1) we have indicated that the expansion starts at the level $1 / c^{2}$, but we could be more precise because the $0 i$ component of $\bar{h}^{\mu \nu}$ starts only at the level $1 / c^{3}$, while the $i j$ component is at least of order $1 / c^{4}$. This does not matter for our purpose; simply in our iteration we include these post-Newtonian coefficients as zero : $\bar{h}_{2}^{0 i}=0$, and $\bar{h}_{2}^{i j}=\bar{h}_{3}^{i j}=0$. For the total stress-energy pseudo-tensor (1.3) we have the same type of expansion,

$$
\bar{\tau}^{\mu \nu}(\mathbf{x}, t, c)=\sum_{n=-2}^{+\infty} \frac{1}{c^{n}} \bar{\tau}_{n}^{\mu \nu}(\mathbf{x}, t, \ln c)
$$

The expansion starts with a term of order $c^{2}$ corresponding to the rest mass-energy of the source $\left(\bar{\tau}^{\mu \nu}\right.$ has the dimension of an energy density). Here we shall always understand the infinite sums such as (3.1)-(3.2) in the sense of formal power series, i.e. merely as an ordered collection of coefficients : e.g. $\left(\begin{array}{l}\bar{h}_{n}^{\mu \nu} \\ n\end{array}\right)_{n \in \mathbb{N}}$. We do not attempt to control the mathematical nature of these series.

In this article we make two important assumptions. First, we assume that the postNewtonian coefficients $\bar{h}_{n}^{\mu \nu}$ (and similarly $\underset{n}{\bar{\tau}^{\mu \nu}}$ ) are smooth functions of space-time,

$$
\bar{h}_{n}^{\mu \nu}(\mathbf{x}, t) \in C^{\infty}\left(\mathbb{R}^{4}\right)
$$

Evidently this comes from our consideration of regular (smooth) extended matter distributions, described by $T^{\mu \nu}(\mathbf{x}, t) \in C^{\infty}\left(\mathbb{R}^{4}\right)$, a priori excluding black holes or point-particle singularities. Second, we assume that the structure of the expansion at spatial infinity, i.e. $r \rightarrow+\infty$ with $t=$ const, is of the type

$$
\forall N \in \mathbb{N}, \quad \bar{h}_{n}^{\mu \nu}=\sum \hat{n}_{L} r^{a}(\ln r)^{p} F_{L, n, a, p}^{\mu \nu}(t)+\mathcal{O}\left(\frac{1}{r^{N}}\right)
$$

(and similarly for each $\bar{\tau}_{n}^{\mu \nu}$ ). On purpose we have written an expansion which is very similar to the one in Eq. (2.14), because as we shall see the functions $F_{L, n, a, p}(t)$ will be equal to 
the post-Newtonian coefficients of the functions $F_{L, a, p}(t)$ appearing in Eq. (2.14). However it is important to realize that in contrast to Eq. (2.14) which is a near-zone expansion $[c f$. the remainder $\mathcal{O}\left(r^{N}\right)$ ], the expansion written in Eq. (3.4) is a far-zone one, with remainder $\mathcal{O}\left(1 / r^{N}\right)$. It would have been clearer to write the latter expansion with some $(\ln r)^{p} / r^{b}$ with $b=-a$, but since we are going to show, from the method of matched asymptotic expansions, that the infinite far-zone expansion (ignoring the remainder) is actually the same as the infinite near-zone expansion, it is better to write it in this form, with the range of the powers of $r$ in Eq. (3.4) being $-a \leq N$ instead of $a \leq N$ in Eq. (2.14). In doing so we are again anticipating on the result of the matching. Finally, we assume that, at any given post-Newtonian order $n$, the maximal divergency of the far-zone expansion (3.4) is finite, i.e. there exists some $a_{\max }(n) \in \mathbb{N}$ such that $a \leq a_{\max }(n)$.

Next we perform the iteration of the post-Newtonian field (3.1) up to any order. Our strategy consists of finding the general post-Newtonian solution of the relaxed Einstein field equation (1.1]). This solution will depend on some arbitrary "homogeneous" solutions, in the form of harmonic solutions solving the source-free d'Alembertian equation (in a perturbative post-Newtonian sense). In a second stage we shall obtain these harmonic solutions by imposing the matching to the external multipolar field obtained in Section II. Finally we shall check that our post-Newtonian solution is divergenceless, i.e. it satisfies the harmoniccoordinate condition (1.2), in consequence of the conservation of the stress-energy pseudo tensor $\tau^{\mu \nu}$. Notice that we do not try to incorporate into the post-Newtonian series the boundary conditions at infinity (viz the no-incoming radiation condition). Indeed this is impossible at the level of the post-Newtonian expansion considered alone, because its validity is limited to the near-zone. Even if we define an "improved" post-Newtonian series by considering some retarded integrals that are formally expanded when $c \rightarrow+\infty$ as series of Poisson-like integrals [11], we ultimately end up with an inconsistency, because the Poissonlike integrals are some local-in-time functionals, depending on the source only at the current time $t$, and we know that the post-Newtonian field starts to depend on the whole past history of the source from the 4PN order 31 33. Therefore, we do not follow this route in 
the present paper, and, instead, we incorporate into the post-Newtonian series the boundary conditions concerning the wave field at infinity by means of the matching equation.

We insert the post-Newtonian ansatz (3.1)-(3.2) into the "relaxed" Einstein field equation (1.1), and equate together the powers of $1 / c$. The result is an infinite set of Poisson-type equations :

$$
\forall n \geq 2, \quad \Delta \bar{h}_{n}^{\mu \nu}=16 \pi G \bar{\tau}_{n-4}^{\mu \nu}+\partial_{t}^{2} \bar{h}_{n-2}^{\mu \nu} .
$$

Evidently, the second term comes from the split of the d'Alembertian operator into a Laplacian and a second time derivative : $\square=\Delta-\frac{1}{c^{2}} \partial_{t}^{2}$; the time derivative $\partial_{0}=\frac{1}{c} \partial_{t}$ is smaller than the spatial gradient $\partial_{i}$ by a factor $1 / c-$ this is the basic tenet of the approximation. When $n=2$ and $n=3$ the second term in Eq. (3.5) is zero, which we take into account by assuming that $\bar{h}_{0}^{\mu \nu}=\bar{h}_{1}^{\mu \nu}=0$. We proceed by induction, i.e. we fix some post-Newtonian order $n$, assume that we succeeded in constructing the sequence of previous coefficients $\bar{h}_{2}^{\mu \nu}, \cdots, \bar{h}_{n-1}^{\mu \nu}$, and from this we infer the next-order coefficient $\bar{h}_{n}^{\mu \nu}$.

The most general solution consists of the sum of a particular solution and of the most general admissible solution of the homogeneous equation, which is simply the source-free Laplace equation. Let us first find a particular solution. We recalled in the introduction that the usual Poisson integral cannot be used to define a solution, because the bound at infinity becomes rapidly divergent when going to higher and higher post-Newtonian orders. Fortunately, thanks to our two assumptions (3.3) and (3.4), we shall be able to define a generalized notion of Poisson integral, in a way similar to our previous definition of a retarded integral operator in Eq. (2.10). That generalized Poisson integral will constitute an appropriate solution of the post-Newtonian equation. For any source term like $\bar{\tau}_{n}^{\mu \nu}$ which is at once smooth, Eq. (3.3), and admits a far-zone expansion of the type (3.4) [note that Eqs. (3.3)-(3.4) hold for $\bar{h}_{n}^{\mu \nu}$ as well as for $\left.\bar{\tau}_{n}^{\mu \nu}\right]$, we multiply it by the same regularization factor as in Eq. (2.10), and then apply the standard Poisson integral. The result, 


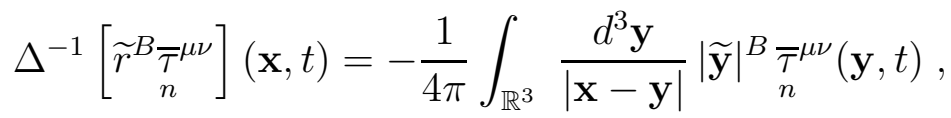

where $|\widetilde{\mathbf{y}}|^{B} \equiv\left|\mathbf{y} / r_{0}\right|^{B}$, defines a certain function of $B \in \mathbb{C}$. The well-definiteness of that integral heavily relies on the behaviour at the bound at infinity. There is no problem with the vicinity of the origin because of the smoothness of the integrand. From the asymptotic expansion (3.4), with $a \leq a_{\max }$ [recall that $\left.a_{\max }=a_{\max }(n)\right]$, we find that the integral converges at infinity when $\Re(B)<-a_{\max }-2$. Next we can prove that the latter function of $B$ generates a (unique) analytic continuation down to a neighbourhood of the origin $B=0$, except at $B=0$ itself, at which value it admits a Laurent expansion with multiple poles up to some finite order. More details are given in Appendix B. Then, we consider the Laurent expansion of that function when $B \rightarrow 0$ and pick up the finite part, or coefficient of the zero-th power of $B$, of that expansion. This defines our generalized Poisson integral :

$$
\widetilde{\Delta^{-1}}\left[\begin{array}{c}
\bar{\tau}_{n}^{\mu \nu} \\
n
\end{array}\right] \underset{B=0}{\mathrm{FP}} \Delta^{-1}\left[\begin{array}{c}
\widetilde{r}^{B} \bar{\tau}^{\mu \nu} \\
n
\end{array}\right]
$$

The finite-part symbol $\underset{B=0}{\mathrm{P}}$ has exactly the same meaning as in Eq. (2.10). However, notice that in contrast to Eq. (2.10) where the regularization factor $\widetilde{r}^{B}$ dealt with the singularity when $r \rightarrow 0$, and hence supposes initially that $\Re(B)$ is a large positive number, in Eq. (3.7) the regularization concerns the behaviour of the integral when $r \rightarrow+\infty$, and so one must start with the situation where $\Re(B)$ is a large negative number. The main properties of our generalized Poisson operator is that it solves the Poisson equation,

$$
\Delta\left[\widetilde{\Delta^{-1}} \bar{\tau}_{n}^{\mu \nu}\right]=\bar{\tau}_{n}^{\mu \nu}
$$

and that the solution $\widetilde{\Delta^{-1}} \bar{\tau}_{n}^{\mu \nu}$ owns the same properties as the source $\bar{\tau}_{n}^{\mu \nu}$, i.e. the smoothness, Eq. (3.3), and the particular far-zone expansion given by Eq. (3.4). These facts are proved in Appendix B. Therefore, we have found a particular solution of the Poisson equation, and, 
furthermore, this solution can be iterated at will, because the operator $\widetilde{\Delta^{-1}}$ keeps the same properties from the source to the corresponding solution. Quite naturally we denote the iterated Poisson operator $\widetilde{\Delta^{-k-1}} \equiv\left(\widetilde{\Delta^{-1}}\right)^{k+1}$; it is not difficult to show that

$$
\widetilde{\Delta^{-k-1}}\left[\bar{\tau}_{n}^{\mu \nu}\right](\mathbf{x}, t)=-\frac{1}{4 \pi} \underset{B=0}{\mathrm{FP}} \int_{\mathbb{R}^{3}} d^{3} \mathbf{y} \frac{|\mathbf{x}-\mathbf{y}|^{2 k-1}}{(2 k) !}|\widetilde{\mathbf{y}}|_{n}^{B} \bar{\tau}_{n}^{\mu \nu}(\mathbf{y}, t) .
$$

From that integral we obtain the operator of the "instantaneous" potentials exactly in the same way as in Eq. (2.20), but now acting on post-Newtonian coefficients such as $\bar{\tau}_{n}^{\mu \nu}$, i.e. satisfying both Eqs. (3.3) and (3.4) :

$$
\widetilde{\mathcal{I}^{-1}}\left[\bar{\tau}_{n}^{\mu \nu}\right]=\sum_{k=0}^{+\infty}\left(\frac{\partial}{c \partial t}\right)^{2 k} \widetilde{\Delta^{-k-1}}\left[\bar{\tau}_{n}^{\mu \nu}\right]
$$

It is clear that we have a particular solution of d'Alembert's equation :

$$
\square\left[\widetilde{\mathcal{I}^{-1}} \bar{\tau}_{n}^{\mu \nu}\right]=\bar{\tau}_{n}^{\mu \nu}
$$

We can check that the definition we have proposed in Eqs. (2.20)-(2.21) is a particular case of the more general definition (3.9)-(3.10). Indeed, if we apply the formulas (3.9)-(3.10) to one of the terms composing the "far-zone" expansion of the post-Newtonian coefficient, i.e. $\hat{n}_{L} r^{a}(\ln r)^{p} F(t)$, we get the same result as the one resulting from Eqs. (2.20)-(2.21).

By means of the Poisson operator $\widetilde{\Delta^{-1}}$ so constructed we first find a particular solution of Eq. (3.5) :

$$
\left(\begin{array}{l}
\bar{h}_{n}^{\mu \nu} \\
n
\end{array}\right)_{\text {part }}=16 \pi G \widetilde{\Delta^{-1}} \bar{\tau}_{n-4}^{\mu \nu}+\partial_{t}^{2}{\widetilde{\Delta^{-1}}}_{n-2}^{\mu \nu} .
$$

To this solution we add the most general solution of the homogeneous Laplace equation. It can be written, using the STF language, as the sum of two multipolar series, one of them being of the type $\hat{x}_{L}$, that is regular at the origin $r=0$, the other one being like $\hat{\partial}_{L}(1 / r)$, 
i.e. regular "at infinity" $r \rightarrow+\infty$ (see Ref. [49] for the notation). Imposing the smoothness condition (3.3) for the post-Newtonian field we discard the second type $\sim \hat{\partial}_{L}(1 / r)$, and retain as the only admissible homogeneous solution the first type $\sim \hat{x}_{L}$. Therefore, we find

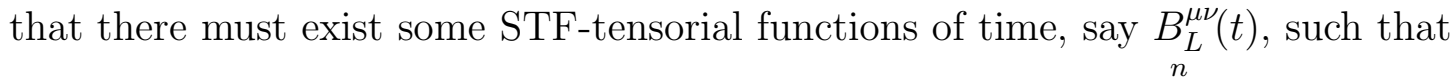

$$
\left(\begin{array}{l}
\bar{h}^{\mu \nu} \\
n
\end{array}\right)_{\mathrm{hom}}=\sum_{l=0}^{+\infty} B_{L}^{\mu \nu}(t) \hat{x}_{L}
$$

The functions $B_{L}^{\mu \nu}(t)$ will be associated with the reaction of the field onto the source, and depend on which boundary conditions are to be imposed on the gravitational field at infinity. The most general solution for the $n$-th post-Newtonian coefficient thus reads

$$
\bar{h}_{n}^{\mu \nu}=\left(\begin{array}{l}
\bar{h}_{n}^{\mu \nu} \\
n
\end{array}\right)_{\mathrm{part}}+\left(\begin{array}{l}
\bar{h}_{n}^{\mu \nu} \\
n_{\mathrm{hom}}
\end{array}\right.
$$

It is now trivial to iterate the process. We substitute for $\bar{h}_{n-2}^{\mu \nu}$ in the right-hand-side of Eq. (3.12) the same expression but with $n$ replaced by $n-2$, and similarly descend untill we stop at either one of the coefficients $\bar{h}_{0}^{\mu \nu}=0$ or $\bar{h}_{1}^{\mu \nu}=0$. At this point $\bar{h}_{n}^{\mu \nu}$ is expressed in

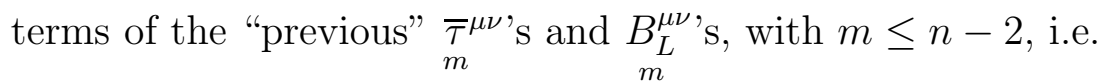

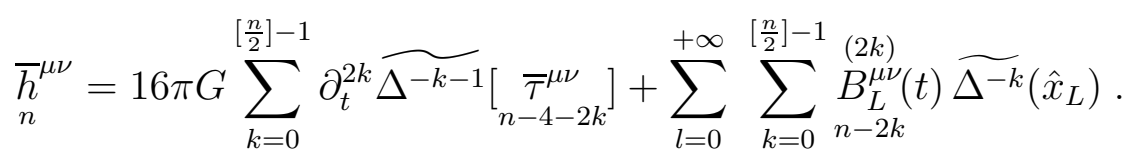

Here $[n / 2]$ denotes the integer part of $n / 2 ; \partial_{t}^{2 k}$ means the $2 k$-th partial time derivative $(\partial / \partial t)^{2 k}$ and the superscript $(2 k)$ the $2 k$-th total time derivative; the operator $\widetilde{\Delta^{-k-1}}$ is the one defined by Eq. (3.9); the object $\widetilde{\Delta^{-k}}\left(\hat{x}_{L}\right)$ has already been introduced in Eq. (2.23). Once we have the result (3.15), we "re-sum" it from $n=2$ up to infinity. After commuting the summations over $n$ and $k$ we arrive at

$$
\bar{h}^{\mu \nu}=\frac{16 \pi G}{c^{4}} \widetilde{\mathcal{I}^{-1}}\left[\bar{\tau}^{\mu \nu}\right]+\sum_{l=0}^{+\infty} \sum_{k=0}^{+\infty} \frac{1}{c^{2 k}} B_{L}^{(2 k)}(t) \widetilde{\Delta^{-k}}\left(\hat{x}_{L}\right)
$$


where we have recognized the operator of the "instantaneous" potentials as defined by Eq. (3.10), and where the functions $B_{L}^{\mu \nu}$ read

$$
B_{L}^{\mu \nu}(t)=\sum_{n=2}^{+\infty} \frac{1}{c^{n}} B_{n}^{\mu \nu}(t) .
$$

A more compact alternative form is

$$
\bar{h}^{\mu \nu}=\frac{16 \pi G}{c^{4}} \widetilde{\mathcal{I}^{-1}}\left[\bar{\tau}^{\mu \nu}\right]+\sum_{l=0}^{+\infty} \Delta \widetilde{\mathcal{I}^{-1}}\left[B_{L}^{\mu \nu}(t) \hat{x}_{L}\right] .
$$

Actually the latter forms are not the best for our purpose. Since the first term in Eqs. (3.16) or (3.18) is a particular solution of the d'Alembert equation [see Eq. (3.11)], the second term is necessarily equal to (the near-zone re-expansion of) a homogeneous solution of the source-free wave equation, and most importantly a regular solution at it. So it should be in the form of some anti-symmetric multipolar waves : retarded minus advanced. Indeed, this readily follows from the second equality in Eq. (2.22). We introduce a new definition $A_{L}^{\mu \nu}(t)$ by posing

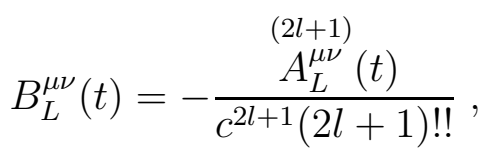

where the $l$-dependent factor is chosen to match with Eq. (2.22). [Because of our assumption of stationarity in the past, $t \leq-\mathcal{T}$, the relation (3.19) determines $A_{L}^{\mu \nu}(t)$ up to a constant. However it is clear that this constant will cancel out in the anti-symmetric wave in Eq. (3.20).] In terms of this definition, we find the final result of this section,

$$
\bar{h}^{\mu \nu}=\frac{16 \pi G}{c^{4}} \widetilde{\mathcal{I}^{-1}}\left[\bar{\tau}^{\mu \nu}\right]+\sum_{l=0}^{+\infty} \hat{\partial}_{L}\left\{\frac{\overline{A_{L}^{\mu \nu}(t-r / c)-A_{L}^{\mu \nu}(t+r / c)}}{2 r}\right\},
$$

where we recall that the overline means the post-Newtonian or equivalently near-zone expansion [see Eq. (2.22)]. For the time being we shall refer to the $A_{L}^{\mu \nu}(t)$ 's as the radiation-reaction functions. 


\section{B. Multipole expansion of the post-Newtonian solution}

In the previous section we obtained the general solution for the post-Newtonian expansion in the form (3.20), and parametrized by some (for the moment) unknown radiation-reaction functions $A_{L}^{\mu \nu}(t)$. To arrive at this we made an assumption concerning the particular structure for the far-zone expansion, at spatial infinity, of the post-Newtonian coefficients : Eq. (3.4). Here we shall denote the corresponding infinite expansion (without remainder term) by means of the same calligraphic letter $\mathcal{M}$ as used to denote the multipole expansion, because the far-zone expansion of the post-Newtonian coefficients is equivalent to a multipolar decomposition. From Eq. (3.4) we have

$$
\mathcal{M}\left(\bar{h}_{n}^{\mu \nu}\right)=\sum \hat{n}_{L} r^{a}(\ln r)^{p} F_{L, n, a, p}^{\mu \nu}(t)
$$

So, summing up the post-Newtonian series,

$$
\mathcal{M}\left(\bar{h}^{\mu \nu}\right)=\sum \hat{n}_{L} r^{a}(\ln r)^{p} F_{L, a, p}^{\mu \nu}(t)
$$

where the functions involved are $F_{L, a, p}(t)=\sum_{n=2}^{+\infty} \frac{1}{c^{n}} F_{L, n, a, p}(t)$. As we can see, the far-zone expansion that we have just postulated is exactly the same, with the same functions $F_{L, a, p}(t)$, as the near-zone expansion we had previously written in Eq. (2.16). This equality is already the matching equation between the near-zone expansion of the multipolar field, $\overline{\mathcal{M}\left(h^{\mu \nu}\right)}$, and the multipolar-far-zone expansion of the post-Newtonian field, $\mathcal{M}\left(\bar{h}^{\mu \nu}\right)$, whose consequences will be investigated in Section [V].

The fundamental result which is needed for computing the far-zone expansion of the postNewtonian series concerns the expansion of the generalized integral operator $\widetilde{\mathcal{I}^{-1}}$ acting on the post-Newtonian source $\bar{\tau}^{\mu \nu}$. More precisely, we are interested in knowing under which

conditions one can commute $\widetilde{\mathcal{I}^{-1}}$ with the operation $\mathcal{M}$ of taking the far-zone expansion. Clearly, the two operations can be commuted at the price of adding some homogeneous 
solution of the d'Alembert equation. We prove in Appendix 9 that the latter homogeneous solution is made of multipolar waves of the symmetric type, i.e. retarded plus advanced. We obtain

$$
\mathcal{M}\left(\widetilde{\mathcal{I}^{-1}}\left[\bar{\tau}^{\mu \nu}\right]\right)=\widetilde{\mathcal{I}^{-1}}\left[\mathcal{M}\left(\bar{\tau}^{\mu \nu}\right)\right]-\frac{1}{4 \pi} \sum_{l=0}^{+\infty} \frac{(-)^{l}}{l !} \hat{\partial}_{L}\left\{\frac{\overline{\mathcal{F}_{L}^{\mu \nu}(t-r / c)+\mathcal{F}_{L}^{\mu \nu}(t+r / c)}}{2 r}\right\} .
$$

Here the overline notation has the same meaning as in Section $\mathbb{I}$ : this is the Taylor expansion when $r \rightarrow 0$, but that expansion should be considered, a posteriori, as an expansion when $r \rightarrow+\infty$. That is, our notation means

$$
\begin{aligned}
\hat{\partial}_{L}\left\{\frac{\overline{\mathcal{F}_{L}^{\mu \nu}(t-r / c)+\mathcal{F}_{L}^{\mu \nu}(t+r / c)}}{2 r}\right\} & =\sum_{i=0}^{+\infty} \frac{\hat{\partial}_{L}\left(r^{2 i-1}\right)}{(2 i) !} \frac{\mathcal{F}^{\mu \nu}(t)}{c^{2 i}} \\
& =\sum_{i=0}^{+\infty} \widetilde{\Delta^{-i}}\left[\hat{\partial}_{L}\left(r^{-1}\right)\right] \frac{\mathcal{F}^{\mu \nu}(t)}{c^{2 i}}
\end{aligned}
$$

[see also Eq. (C14)] where the right-hand-sides are to be considered as some expansions at spatial infinity, of the general type given by Eq. (3.22). The functions $\mathcal{F}_{L}^{\mu \nu}(t)$ parametrizing these symmetric waves are STF and explicitly given by

$$
\mathcal{F}_{L}^{\mu \nu}(t)=\sum_{j=0}^{+\infty} \frac{1}{c^{2 j}} \underset{B=0}{\mathrm{FP}} \int d^{3} \mathbf{x}|\widetilde{\mathbf{x}}|^{B} \widetilde{\Delta^{-j}}\left[\hat{x}_{L}\right] \partial_{t}^{2 j} \bar{\tau}^{\mu \nu}(\mathbf{x}, t)
$$

where $\widetilde{\Delta^{-j}}\left[\hat{x}_{L}\right]$ is given by Eq. (2.23). See the proof in Appendix Q9. An alternative form reads as

$$
\mathcal{F}_{L}^{\mu \nu}(t)=\underset{B=0}{\mathrm{FP}} \int d^{3} \mathbf{x}|\widetilde{\mathbf{x}}|^{B} \hat{x}_{L} \overline{\int_{-1}^{1} d z \delta_{l}(z) \bar{\tau}^{\mu \nu}(\mathbf{x}, t-z|\mathbf{x}| / c)},
$$

where the integration over the $z$-dependent cone $t-z|\mathbf{y}| / c$ involves a weighting function $\delta_{l}(z)$ that is closely related to the function $\gamma_{l}(z)$ introduced in Eq. (2.25) :

$$
\delta_{l}(z)=\frac{(2 l+1) ! !}{2^{l+1} l !}\left(1-z^{2}\right)^{l}=-\frac{1}{2} \gamma_{l}(z)
$$


and whose integral is normalized to one : $\int_{-1}^{1} d z \delta_{l}(z)=1$ t . The function $\delta_{l}(z)$ approaches the Dirac delta-function in the limit of large $l: \lim _{l \rightarrow+\infty} \delta_{l}(z)=\delta(z)$. In Eq. (3.26) we have indicated by means of an overline the fact that this expression is valid only in a sense of post-Newtonian expansion. Note that because the latter post-Newtonian expansion is "even", containing only even powers of $1 / c$, one can replace the argument $t-z|\mathbf{y}| / c$ inside $\mathcal{F}_{L}^{\mu \nu}(t)$ equivalently by $t+z|\mathbf{y}| / c$.

Finally, thanks to Eqs. (3.23)-(3.27), we are in a position to write the infinite multipolarfar-zone expansion of the post-Newtonian solution as

$$
\begin{aligned}
\mathcal{M}\left(\bar{h}^{\mu \nu}\right) & =\frac{16 \pi G}{c^{4}} \widetilde{\mathcal{I}^{-1}}\left[\mathcal{M}\left(\bar{\tau}^{\mu \nu}\right)\right] \\
& -\frac{4 G}{c^{4}} \sum_{l=0}^{+\infty} \frac{(-)^{l}}{l !} \hat{\partial}_{L}\left\{\frac{\overline{\mathcal{F}_{L}^{\mu \nu}(t-r / c)+\mathcal{F}_{L}^{\mu \nu}(t+r / c)}}{2 r}\right\} \\
& +\sum_{l=0}^{+\infty} \hat{\partial}_{L}\left\{\frac{\overline{A_{L}^{\mu \nu}(t-r / c)-A_{L}^{\mu \nu}(t+r / c)}}{2 r}\right\}
\end{aligned}
$$

We recall that the radiation-reaction functions $A_{L}^{\mu \nu}(t)$ are still undetermined at this stage. The symmetric and anti-symmetric waves are given by Eqs. (3.24) and (2.22) respectively, considered here as infinite far-zone expansions.

\section{MATCHING}

In Section [IA, we found the most general expression for the multipolar expansion $\mathcal{M}\left(h^{\mu \nu}\right)$, satisfying the no-incoming radiation condition, in terms of some unknown "multipole-moment" STF-functions $X_{L}^{\mu \nu}(t)$ [see Eq. (2.15)]. On the other hand, in Section ПIIA, we obtained the most general solution for the post-Newtonian expansion $\bar{h}^{\mu \nu}$,

\footnotetext{
${ }^{4}$ The normalization for the function $\delta_{l}(z)$ is consistent with that of the function $\gamma_{l}(z)$ : $\int_{1}^{+\infty} d z \gamma_{l}(z)=1$, owing to the fact that the integral $\int_{-\infty}^{+\infty} d z\left(1-z^{2}\right)^{l}$ is zero by complex analytic continuation in $l \in \mathbb{C}$.
} 
as parametrized by a set of unknown "radiation-reaction" STF-functions $A_{L}^{\mu \nu}(t)$ [see Eq. $(3.20)]$. We are now imposing the matching condition

$$
\overline{\mathcal{M}\left(h^{\mu \nu}\right)} \equiv \mathcal{M}\left(\bar{h}^{\mu \nu}\right) .
$$

In fact we have already postulated this equation when writing that the two formal expansions (2.16) and (3.22) are the same. Recall that the matching equation (4.1) results from the numerical equality $\mathcal{M}\left(h^{\mu \nu}\right)=\bar{h}^{\mu \nu}$, verified in the exterior near-zone : $a<r \ll \lambda$. It is physically justified only for post-Newtonian sources, for which the exterior near-zone exists. The matching equation is actually a functional identity, i.e. true $\forall(\mathbf{x}, t) \in \mathbb{R}_{*}^{3} \times \mathbb{R}$; it identifies, term-by-term, two asymptotic singular expansions, each of them being formally taken outside its own domain of validity. In the present context, the matching equation insists that the infinite near-zone expansion, $r \rightarrow 0$, of the exterior multipolar field is identical to the infinite far-zone expansion, $r \rightarrow+\infty$, of the inner post-Newtonian field. Let us show now that Eq. (4.1) permits determining all the unknowns of the problem : i.e., at once, the multipole moments $X_{L}^{\mu \nu}$ and the radiation-reaction functions $A_{L}^{\mu \nu}$. In particular we find that the multipole moments $X_{L}^{\mu \nu}$ are in agreement with the earlier result derived in Ref. 40].

For the sake of clarity we re-state here the two results we reached for the two sides of Eq. (4.1). The left-hand-side was obtained in Eq. (2.26) :

$$
\begin{aligned}
\overline{\mathcal{M}\left(h^{\mu \nu}\right)} & =\widetilde{\mathcal{I}^{-1}}\left[\overline{\mathcal{M}\left(\Lambda^{\mu \nu}\right)}\right] \\
& -\frac{4 G}{c^{4}} \sum_{l=0}^{+\infty} \frac{(-)^{l}}{l !} \hat{\partial}_{L}\left\{\frac{\overline{\mathcal{R}_{L}^{\mu \nu}(t-r / c)-\mathcal{R}_{L}^{\mu \nu}(t+r / c)}}{2 r}\right\} \\
& +\sum_{l=0}^{+\infty} \hat{\partial}_{L}\left\{\frac{\overline{X_{L}^{\mu \nu}(t-r / c)}}{r}\right\},
\end{aligned}
$$

in which the functions $\mathcal{R}_{L}^{\mu \nu}$, which come from the non-linearities of the field equations in vacuum, are known from Eq. (2.24). The right-hand-side of the matching equation was found in Eq. (3.28) : 


$$
\begin{aligned}
\mathcal{M}\left(\bar{h}^{\mu \nu}\right) & =\frac{16 \pi G}{c^{4}} \widetilde{\mathcal{I}^{-1}}\left[\mathcal{M}\left(\bar{\tau}^{\mu \nu}\right)\right] \\
& -\frac{4 G}{c^{4}} \sum_{l=0}^{+\infty} \frac{(-)^{l}}{l !} \hat{\partial}_{L}\left\{\frac{\overline{\mathcal{F}_{L}^{\mu \nu}(t-r / c)+\mathcal{F}_{L}^{\mu \nu}(t+r / c)}}{2 r}\right\} \\
& +\sum_{l=0}^{+\infty} \hat{\partial}_{L}\left\{\frac{\overline{A_{L}^{\mu \nu}(t-r / c)-A_{L}^{\mu \nu}(t+r / c)}}{2 r}\right\}
\end{aligned}
$$

Here, the functions $\mathcal{F}_{L}^{\mu \nu}$, which depend on the matter and gravitational content of the postNewtonian source, take the definite expression given by Eqs. (3.25)-(3.26).

Comparing the equations (4.2) and (4.3), we readily discover that they share an obvious common term, that is the first one. Indeed, we manifestly have

$$
\widetilde{\mathcal{I}^{-1}}\left[\overline{\mathcal{M}\left(\Lambda^{\mu \nu}\right)}\right]=\widetilde{\mathcal{I}^{-1}}\left[\mathcal{M}\left(\bar{\Lambda}^{\mu \nu}\right)\right]=\frac{16 \pi G}{c^{4}} \widetilde{\mathcal{I}^{-1}}\left[\mathcal{M}\left(\bar{\tau}^{\mu \nu}\right)\right]
$$

The first equality comes from the matching equation, as applied to the gravitational source term $\Lambda^{\mu \nu}$, and the second equality comes from the fact that the matter tensor $T^{\mu \nu}$ has a compact support, so that $\mathcal{M}\left(T^{\mu \nu}\right)=0$. Hence the two first terms in Eqs. (4.2) and (4.3) match together. This is a somewhat remarkable fact, because most of the complexity of the Einstein field equations is actually contained into these terms, either $\mathcal{I}^{-1}\left[\overline{\mathcal{M}\left(\Lambda^{\mu \nu}\right)}\right]$ for the external field or $\frac{16 \pi G}{c^{4}} \mathcal{I}^{-1}\left[\mathcal{M}\left(\bar{\tau}^{\mu \nu}\right)\right]$ for the inner one. But for doing the matching we don't need all this complexity; these two terms match and therefore are to be identified. Notice also that this is a non-trivial result, since the two sides of Eq. (4.1) strongly depend on the yet unknown functions $A_{L}^{\mu \nu}$ and $X_{L}^{\mu \nu}$, which enter the latter two terms in a very intricate way, coupled together as they are via the non-linearities of the field equations. Nevertheless, the matching equation tells us that these terms must be rigorously identical.

As soon as we have noticed that the first terms in Eqs. (4.2) and (4.3) are equal, we can compare the other ones, and because the retarded and advanced waves have some different structures, they must be matched independently, so we get two relations to be satisfied. We find that these are solved if and only if the multipole-moments in the exterior field and the radiation-reaction functions in the inner field are given by 


$$
\begin{aligned}
& X_{L}^{\mu \nu}(t)=-\frac{4 G}{c^{4}} \frac{(-)^{l}}{l !} \mathcal{F}_{L}^{\mu \nu}(t) \\
& A_{L}^{\mu \nu}(t)=-\frac{4 G}{c^{4}} \frac{(-)^{l}}{l !}\left[\mathcal{F}_{L}^{\mu \nu}(t)+\mathcal{R}_{L}^{\mu \nu}(t)\right] .
\end{aligned}
$$

Therefore, both the multipole-moments and the radiation-reaction terms are determined as some explicit functionals of the pseudo-tensor $\tau^{\mu \nu}$ and nothing else. (Actually we could add any constant to the definition of $A_{L}^{\mu \nu}(t)$, but this is physically irrelevant because the constant disappears from the anti-symmetric waves; see also Ref. [50.)

Finally, by way of summary of the results, we take back the latter expressions and fill in the external and inner fields, which are then entirely determined as coming from a unique solution of the Einstein field equations in harmonic coordinates, valid everywhere inside and outside the source. The exterior field is

$$
\mathcal{M}\left(h^{\mu \nu}\right)=\widetilde{\square_{\text {Ret }}^{-1}}\left[\mathcal{M}\left(\Lambda^{\mu \nu}\right)\right]-\frac{4 G}{c^{4}} \sum_{l=0}^{+\infty} \frac{(-)^{l}}{l !} \hat{\partial}_{L}\left\{\frac{\mathcal{F}_{L}^{\mu \nu}(t-r / c)}{r}\right\},
$$

where the multipole moments are given in terms of the post-Newtonian expansion of the stress-energy pseudo-tensor by

$$
\begin{aligned}
\mathcal{F}_{L}^{\mu \nu}(t) & =\underset{B=0}{\mathrm{FP}} \int d^{3} \mathbf{y}|\widetilde{\mathbf{y}}|^{B} \hat{y}_{L} \widetilde{\int_{-1}^{1} d z \delta_{l}(z) \bar{\tau}^{\mu \nu}(\mathbf{y}, t-z|\mathbf{y}| / c)} \\
& =\sum_{j=0}^{+\infty} \frac{1}{c^{2 j}} \underset{B=0}{\mathrm{FP}} \int d^{3} \mathbf{y}|\widetilde{\mathbf{y}}|^{B} \widetilde{\Delta^{-j}}\left[\hat{y}_{L}\right] \partial_{t}^{2 j} \bar{\tau}^{\mu \nu}(\mathbf{y}, t) .
\end{aligned}
$$

This result is in perfect agreement with the multipole decomposition of the exterior field obtained in Ref. [40] [see Eqs. (3.13)-(3.14) there]. On the other hand, the inner postNewtonian field is given by

$$
\bar{h}^{\mu \nu}=\frac{16 \pi G}{c^{4}} \widetilde{\mathcal{I}^{-1}}\left[\bar{\tau}^{\mu \nu}\right]-\frac{4 G}{c^{4}} \sum_{l=0}^{+\infty} \frac{(-)^{l}}{l !} \hat{\partial}_{L}\left\{\frac{\overline{\mathcal{A}_{L}^{\mu \nu}(t-r / c)-\mathcal{A}_{L}^{\mu \nu}(t+r / c)}}{2 r}\right\}
$$

where the radiation-reaction function is composed of two terms :

$$
\mathcal{A}_{L}^{\mu \nu}(t)=\mathcal{F}_{L}^{\mu \nu}(t)+\mathcal{R}_{L}^{\mu \nu}(t)
$$


The first term is nothing but the exterior multipole moment given by Eq. (4.8), and one can check that it contains the standard radiation-reaction effect at the $2.5 \mathrm{PN}$ order. The $\mathcal{R}_{L}^{\mu \nu}$-term is defined by Eq. (2.24), or, rather, the post-Newtonian expansion of it, i.e.

$$
\mathcal{R}_{L}^{\mu \nu}(t)=\underset{B=0}{\mathrm{FP}} \int d^{3} \mathbf{y}|\widetilde{\mathbf{y}}|^{B} \hat{y}_{L} \overline{\int_{1}^{+\infty} d z \gamma_{l}(z) \mathcal{M}\left(\tau^{\mu \nu}\right)(\mathbf{y}, t-z|\mathbf{y}| / c)} .
$$

This term is quite interesting : it depends on the non-linearities of the exterior field, described by the gravitational source term $\mathcal{M}\left(\tau^{\mu \nu}\right)$ (or, more precisely, the non-stationary part of it [50]), which are to be computed by means of the multipolar-post-Minkowskian algorithm of Refs. [36.32] (see in particular Section III.D in Ref. [32] for some detailed computations of this term). Physically the function $\mathcal{R}_{L}^{\mu \nu}$ contains the effect of wave tails in the radiation reaction force which arises at the $4 \mathrm{PN}$ order [31 33]. It is not difficult [using notably the formula (5.21) below] to derive the more explicit expression for the contribution of $\mathcal{R}_{L}^{\mu \nu}$ to the anti-symmetric wave in Eq. (4.9) :

$$
\begin{aligned}
& \hat{\partial}_{L}\left\{\frac{\overline{\mathcal{R}_{L}^{\mu \nu}(t-r / c)-\mathcal{R}_{L}^{\mu \nu}(t+r / c)}}{2 r}\right\}= \\
& \sum_{i=0}^{l} \frac{(-)^{l}(l+i) !}{2^{i} i !(l-i) !} \sum_{k=0}^{+\infty} \frac{\widetilde{\Delta^{-k}}\left(\hat{x}_{L}\right)}{c^{2 k+l-i}} \underset{B=0}{\mathrm{FP}} \int d^{3} \mathbf{y}|\widetilde{\mathbf{y}}|^{B} \frac{\hat{y}_{L}}{|\mathbf{y}|^{l+i+1}} \partial_{t}^{2 k+l-i} \mathcal{M}\left(\tau^{\mu \nu}\right)(\mathbf{y}, t-|\mathbf{y}| / c) .
\end{aligned}
$$

When they are computed by post-Minkowskian approximations, the remaining integrals will typically yield, after integration over the angles, some "hereditary-like" contributions, depending on the whole integrated past of the matter source (see Ref. [32]).

It is tempting to speculate that the second term in Eq. (4.9), made of the anti-symmetric multipolar waves parametrized by the functions $\mathcal{A}_{L}^{\mu \nu}(t)$, can be regarded as the contribution, in a sense to be made more precise, of the radiation reaction forces at work inside the post-Newtonian source. [Indeed we have checked that these functions contain the known radiation-reaction terms at the dominant $2.5 \mathrm{PN}$ order as well as the dominant contribution of tails at the $4 \mathrm{PN}$ order.] We shall leave for future work the systematic study of this term, as well as the possibility to answer the latter speculation. 


\section{HARMONIC-COORDINATE CONDITION}

The latter solution for the post-Newtonian field, Eqs. (4.9)-(4.12), has been obtained without imposing, in an explicit way, the condition of harmonic coordinates (1.2). Indeed, we have assumed this condition to be true, and we simply matched together the postNewtonian and multipolar-post-Minkowskian expansions, satisfying the relaxed Einstein field equations (1.1) in their respective domains. We found that the matching determines uniquely the expressions of the multipole moments $X_{L}^{\mu \nu}(t)$ and radiation-reaction functions $A_{L}^{\mu \nu}(t)$ as some functionals of the stress-energy pseudo-tensor $\tau^{\mu \nu}$. However, we never used the harmonic-coordinate condition during the matching; it was not necessary for the formal determination of the unknown parameters $\left(X_{L}^{\mu \nu}, A_{L}^{\mu \nu}\right)$. Therefore, it is quite important to check that our post-Newtonian solution is divergenceless as a consequence of the conservation of the pseudo-tensor $\tau^{\mu \nu}$ [see Eq. (1.2)], so that we really grasp a solution of the full Einstein field equations.

We check the divergenceless of $\bar{h}^{\mu \nu}$ directly on Eq. (3.16). We apply the $\partial_{\mu}$ operator on each side of the equality :

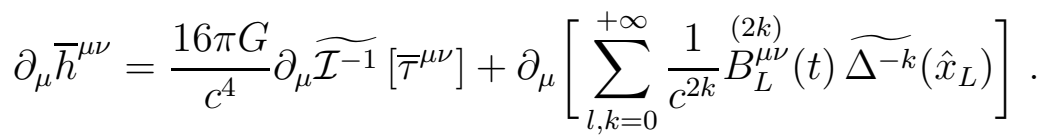

We must transform the two terms on the right-hand-side in order to make explicit the fact that these two terms are exactly the opposite. The first term, that is to say, the divergence of the $\widetilde{\mathcal{I}^{-1}}$ operator, is not obvious since, even if time derivatives commute with $\widetilde{\mathcal{I}^{-1}}$, spatial derivatives do not,

$$
\partial_{\mu} \widetilde{\mathcal{I}-1}\left[\bar{\tau}^{\mu \nu}\right]=\widetilde{\mathcal{I}^{-1}}\left[\partial_{0} \bar{\tau}^{0 \nu}\right]+\partial_{i} \widetilde{\mathcal{I}^{-1}}\left[\bar{\tau}^{i \nu}\right]
$$

$\widetilde{\mathcal{I}^{-1}}$ is a sum of $\widetilde{\Delta^{-k-1}} \partial_{t}^{2 k}$, and spatial derivatives do not commute with $\widetilde{\Delta^{-k-1}}$ because of the $|\widetilde{\mathbf{y}}|^{B}$ factor [see Eq. (3.9) for the exact expression]. To see how to tackle this problem, let us start with the spatial divergence of $\widetilde{\Delta^{-k-1}}$. We assume that $\bar{\tau}(\mathbf{x}, t)$ is a function of the 
"post-Newtonian" type, i.e. satisfies the requirements (3.3)-(3.4). The $\partial_{i}$ derivative, in Eq. (5.3), applies first to the $k^{\text {th }}$-Poisson's kernel but after having noticed that the $x^{i}$-derivative of this kernel was equal to minus the $y^{i}$-derivative of it, we can make an integration by part and distribute the $y^{i}$-derivative on $|\widetilde{\mathbf{y}}|^{B}$ and on $\bar{\tau}$ so that

$$
\begin{aligned}
& \partial_{i} \widehat{\Delta^{-k-1}}(\bar{\tau}(\mathbf{x}, t))=-\frac{1}{4 \pi} \underset{B=0}{\mathrm{FP}} \int d^{3} \mathbf{y}|\widetilde{\mathbf{y}}|^{B} \partial_{x^{i}}\left(\frac{|\mathbf{x}-\mathbf{y}|^{2 k-1}}{(2 k) !}\right) \bar{\tau}(\mathbf{y}, t)= \\
& -\frac{1}{4 \pi} \underset{B=0}{\mathrm{FP}} \int d^{3} \mathbf{y}|\widetilde{\mathbf{y}}|^{B} \frac{|\mathbf{x}-\mathbf{y}|^{2 k-1}}{(2 k) !} \partial_{i} \bar{\tau}(\mathbf{y}, t)-\frac{1}{4 \pi} \underset{B=0}{\mathrm{FP}} \int d^{3} \mathbf{y} \partial_{i}\left(|\widetilde{\mathbf{y}}|^{B}\right) \frac{|\mathbf{x}-\mathbf{y}|^{2 k-1}}{(2 k) !} \bar{\tau}(\mathbf{y}, t) .
\end{aligned}
$$

The first term in the last line of the previous equality is equal to $\widetilde{\Delta^{-k-1}}\left(\partial_{i} \bar{\tau}\right)$. Now, let us concentrate on the last term in the same line. We can, of course, write $\partial_{i}\left(|\widetilde{\mathbf{y}}|^{B}\right)=$ $B n_{i}|\widetilde{\mathbf{y}}|^{B-1} / r_{0}$. Moreover, since $\bar{\tau}(\mathbf{y}, t)$ is regular at the origin $(|\mathbf{y}|=0)$, the integral is always convergent on any neighbourhood of the origin. Translating these two remarks in the last integral of Eq. (5.3), and since we take the finite part when $B=0$, this last integral is zero, because of the explicit factor $B$, when ranging from $|\mathbf{y}|=0$ up to some arbitrary finite value $|\mathbf{y}|=\mathcal{R}$. So, after replacing $|\mathbf{x}-\mathbf{y}|^{2 k-1}$ by $(2 k) ! \widetilde{\Delta^{-k}}\left(|\mathbf{x}-\mathbf{y}|^{-1}\right)$ we are left, in Eq. (5.4), with one integral ranging over $|\mathbf{y}|>\mathcal{R}$,

$$
\begin{aligned}
& \underset{B=0}{\mathrm{FP}} \int d^{3} \mathbf{y} \partial_{i}\left(|\widetilde{\mathbf{y}}|^{B}\right) \frac{|\mathbf{x}-\mathbf{y}|^{2 k-1}}{(2 k) !} \bar{\tau}(\mathbf{y}, t) \\
& =\underset{B=0}{\mathrm{FP}} \int_{|\mathbf{y}|>\mathcal{R}} d^{3} \mathbf{y} \partial_{i}\left(|\widetilde{\mathbf{y}}|^{B}\right) \mathcal{M}\left[\widetilde{\Delta^{-k}}\left(|\mathbf{x}-\mathbf{y}|^{-1}\right)\right] \mathcal{M}(\bar{\tau})(\mathbf{y}, t) \\
& =\sum_{n=0}^{k} \sum_{l \geq 0} \frac{(-)^{l}}{l !} \widetilde{\Delta^{-k+n}}\left(\hat{x}_{L}\right) \underset{B=0}{\mathrm{FP}} \int_{|\mathbf{y}|>\mathcal{R}} d^{3} \mathbf{y} \partial_{i}\left(|\widetilde{\mathbf{y}}|^{B}\right) \widetilde{\Delta^{-n}}\left(\hat{\partial}_{L}\left(|\mathbf{y}|^{-1}\right)\right) \mathcal{M}(\bar{\tau})(\mathbf{y}, t) .
\end{aligned}
$$

In the last line of the previous equation, we expanded the $k^{\text {th }}$-Poisson's kernel for $|\mathbf{y}| \gg|\mathbf{x}|$ using Eq. (C12). This is possible thanks to the fact that $\mathcal{R}$ is arbitrary and may be chosen such that $\mathcal{R} \gg|\mathbf{x}|$. We also note that $\bar{\tau}$ turned into $\mathcal{M}(\bar{\tau})$ because $\bar{\tau}=\mathcal{M}(\bar{\tau})$ in the far zone. In this way,

$$
\begin{aligned}
& \partial_{i} \widehat{\Delta^{-1-k}}[\bar{\tau}(\mathbf{x}, t)]=\widetilde{\Delta^{-1-k}}\left[\partial_{i} \bar{\tau}(\mathbf{x}, t)\right] \\
& -\frac{1}{4 \pi} \sum_{n=0}^{k} \sum_{l \geq 0} \frac{(-)^{l}}{l !} \widetilde{\Delta^{-k+n}}\left(\hat{x}_{L}\right) \underset{B=0}{\mathrm{FP}} \int_{|\mathbf{y}|>\mathcal{R}} d^{3} \mathbf{y} \partial_{i}\left(|\widetilde{\mathbf{y}}|^{B}\right) \widetilde{\Delta^{-n}}\left(\hat{\partial}_{L}\left(|\mathbf{y}|^{-1}\right)\right) \mathcal{M}(\bar{\tau})(\mathbf{y}, t)
\end{aligned}
$$


and we notice that the commutation of the spatial derivative and the generalized $k^{\text {th }}$-Poisson integral depends only on the behaviour of $\bar{\tau}(\mathbf{x}, t)$ at spatial infinity. This fact was foreseeable since for a function $\bar{\tau}(\mathbf{x}, t)$ with compact support the commutation would be trivial. Thanks to the general result given by Eq. (5.5), in which we replace $\bar{\tau}$ by $\bar{\tau}^{i \nu}$, we can determine the spatial divergence of $\widetilde{\mathcal{I}^{-1}}\left(\bar{\tau}^{i \nu}\right)$. We can then get $\partial_{\mu} \widetilde{\mathcal{I}^{-1}}\left[\bar{\tau}^{\mu \nu}\right]$ that is the sum of $\widetilde{\mathcal{I}^{-1}}\left[\partial_{\mu} \bar{\tau}^{\mu \nu}\right]$ and a non-trivial term. Since $\partial_{\mu} \bar{\tau}^{\mu \nu}=0$, the result for the first term of Eq. (5.1) reduces to the non-trivial term, that is to say :

$$
\begin{aligned}
& \frac{16 \pi G}{c^{4}} \partial_{\mu} \widetilde{\mathcal{I}^{-1}}\left[\bar{\tau}^{\mu \nu}\right]
\end{aligned}
$$

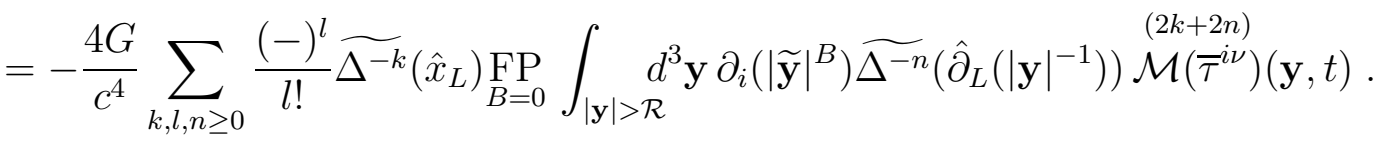

Now, we want to prove that the second term in the right-hand-side of Eq. (5.1) is exactly the opposite. In Eq. (5.7), we expand this last term in its $3+1$ form so that we can treat separately terms with time derivative and terms with spatial derivative,

$$
\sum_{n, l \geq 0} \frac{1}{c^{2 n}} \stackrel{(2 n)}{B_{L}^{i \nu}}(t) \partial_{i}\left[\widetilde{\Delta^{-n}}\left(\hat{x}_{L}\right)\right]+\sum_{n, l \geq 0} \frac{1}{c^{2 n}} \partial_{0} B_{L}^{0 \nu}(t) \widetilde{\Delta^{-n}}\left(\hat{x}_{L}\right)
$$

The first term of Eq. (5.7), thanks to a STF formula, can be written without the use of spatial derivative. The index $i$ coming from this derivative is distributed on the multi-index $L$ in the way

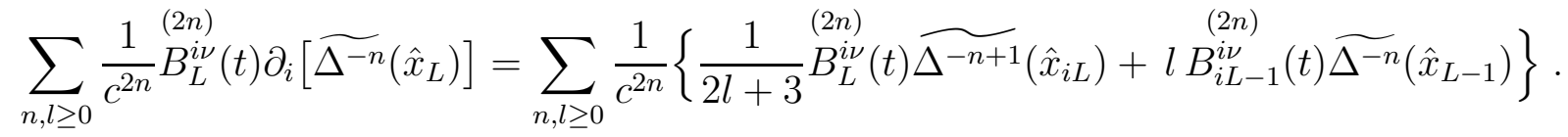

In the second term of Eq. (5.7), we express the function $B_{L}^{0 \nu}$ in terms of $\mathcal{F}_{L}^{0 \nu}$ and $\mathcal{R}_{L}^{0 \nu}[c f$. Eqs. (3.19) and (4.6)] because the time derivative, $\partial_{0}$, will act on the integrand of these two time-varying moments :

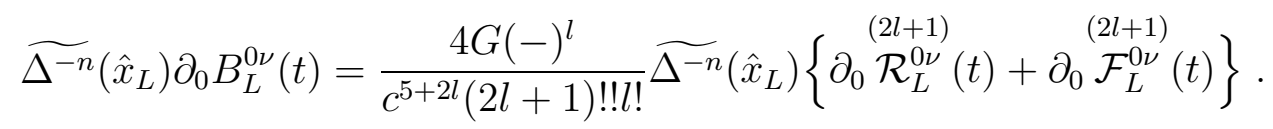

First, we investigate the case of $\partial_{0} \mathcal{F}_{L}^{0 \nu}$, using the formula (3.25), where the time derivative $\operatorname{acts}$ on $\bar{\tau}^{0 \nu}$, 


$$
\widetilde{\Delta^{-n}}\left(\hat{x}_{L}\right) \partial_{0} \mathcal{F}_{L}^{0 \nu}(t)=\widetilde{\Delta^{-n}}\left(\hat{x}_{L}\right) \sum_{k \geq 0} \frac{1}{c^{2 k}} \mathrm{FP}_{B=0} \int d^{3} \mathbf{y}|\widetilde{\mathbf{y}}|^{B} \widetilde{\Delta^{-k}}\left(\hat{y}_{L}\right) \partial_{0} \bar{\tau}^{0 \nu}(\mathbf{y}, t) .
$$

We can replace $\partial_{0} \bar{\tau}^{0 \nu}$ by $-\partial_{i} \bar{\tau}^{i \nu}$ thanks to the conservation equation of the pseudo-tensor. After integrating by part we get

$$
\begin{aligned}
& \widetilde{\Delta^{-n}}\left(\hat{x}_{L}\right) \sum_{k \geq 0} \frac{1}{c^{2 k}} \underset{B=0}{\mathrm{FP}} \int d^{3} \mathbf{y} \partial_{i}\left(|\widetilde{\mathbf{y}}|^{B}\right) \widetilde{\Delta^{-k}}\left(\hat{y}_{L}\right) \bar{\tau}^{i \nu}(\mathbf{y}, t) \\
& +\widetilde{\Delta^{-n}}\left(\hat{x}_{L}\right) \sum_{n \geq 0} \frac{1}{c^{2 k}} \underset{B P}{\mathrm{FP}} \int d^{3} \mathbf{y}|\widetilde{\mathbf{y}}|^{B} \partial_{i}\left(\widetilde{\Delta^{-k}}\left(\hat{y}_{L}\right)\right) \bar{\tau}^{i \nu}(\mathbf{y}, t) .
\end{aligned}
$$

The same STF formula as used in Eq. (5.8) enables one to transform the second term of Eq. (5.11) so that, at the end, we get the definitive result :

$$
\begin{aligned}
\widetilde{\Delta^{-n}}\left(\hat{x}_{L}\right) \partial_{0} \mathcal{F}_{L}^{0 \nu}(t)= & \widetilde{\Delta^{-n}}\left(\hat{x}_{L}\right) \sum_{k \geq 0} \frac{1}{c^{2 k}} \underset{B=0}{\mathrm{FP}} \int d^{3} \mathbf{y} \partial_{i}\left(|\widetilde{\mathbf{y}}|^{B}\right) \widetilde{\Delta^{-k}}\left(\hat{y}_{L}\right) \bar{\tau}^{i \nu}(\mathbf{y}, t) \\
& +l \widetilde{\Delta^{-n}}\left(\hat{x}_{i L-1}\right) \mathcal{F}_{L-1}^{i \nu}(t)+\frac{\widetilde{\Delta^{-n}}\left(\hat{x}_{L}\right)}{c^{2}(2 l+3)} \mathcal{F}_{i L}^{i \nu}(t) .
\end{aligned}
$$

We can, now, investigate the case of the first term in Eq. (5.9), which is a little bit more complicated since it involves a retarded integral,

$\widetilde{\Delta^{-n}}\left(\hat{x}_{L}\right) \partial_{0} \mathcal{R}_{L}^{0 \nu}(t)=\widetilde{\Delta^{-n}}\left(\hat{x}_{L}\right) \underset{B=0}{\operatorname{FP}} \int d^{3} \mathbf{y}|\widetilde{\mathbf{y}}|^{B} \hat{y}_{L} \int_{1}^{\infty} d z \gamma_{l}(z) \mathcal{M}\left(\partial_{0} \tau^{0 \nu}\right)(\mathbf{y}, t-z|\mathbf{y}| / c)$,

where the function $\gamma_{l}(z)$ is given by Eq. (2.25) (for simplicity's sake we do not write the overline indicating the post-Newtonian expansion). We do the replacement of $\partial_{0} \tau^{0 \nu}$ into $-\partial_{i} \tau^{i \nu}$. Before integrating by part, we better have to notice that the partial derivative $\partial_{i}$ acts on $\tau^{i \nu}$ which is then evaluated at the event $(\mathbf{y}, t-z|\mathbf{y}| / c)$; we must be careful about the space dependence of the time variable $t-z|\mathbf{y}| / c$. The last equation then becomes

$$
\begin{aligned}
& -\widetilde{\Delta^{-n}}\left(\hat{x}_{L}\right) \underset{B=0}{\operatorname{FP}} \int d^{3} \mathbf{y}|\widetilde{\mathbf{y}}|^{B} \hat{y}_{L} \partial_{i}\left(\int_{1}^{\infty} d z \gamma_{l}(z) \mathcal{M}\left(\tau^{i \nu}\right)(\mathbf{y}, t-z|\mathbf{y}| / c)\right) \\
& -\widetilde{\Delta^{-n}}\left(\hat{x}_{L}\right) \underset{B=0}{\operatorname{FP}} \int d^{3} \mathbf{y}|\widetilde{\mathbf{y}}|^{B} \hat{y}_{L} n_{i} \int_{1}^{\infty} d z \frac{z}{c} \gamma_{l}(z) \mathcal{M}\left(\tau^{i \nu}\right)(\mathbf{y}, t-z|\mathbf{y}| / c) .
\end{aligned}
$$

In this way, the first term can be integrated by part straigthforwardly, in terms of $d^{3} \mathbf{y}$ integration, showing up a $\partial_{i}\left(|\widetilde{\mathbf{y}}|^{B}\right)$ term and a $\partial_{i}\left(\hat{y}_{L}\right)$ term. The second term will also be 
integrated by part, in terms of $d z$ integration, using the fact $\frac{d}{d z}\left[\gamma_{l+1}(z)\right]=-(2 l+3) z \gamma_{l}(z)$; so we have

$$
\begin{aligned}
& \widetilde{\Delta^{-n}}\left(\hat{x}_{L}\right) \underset{B=0}{\mathrm{FP}} \int d^{3} \mathbf{y} \partial_{i}\left(|\widetilde{\mathbf{y}}|^{B}\right) \hat{y}_{L} \int_{1}^{\infty} d z \gamma_{l}(z) \mathcal{M}\left(\tau^{i \nu}\right)(\mathbf{y}, t-z|\mathbf{y}| / c) \\
& +\widetilde{\Delta^{-n}}\left(\hat{x}_{L}\right) \underset{B=0}{\operatorname{FP}} \int d^{3} \mathbf{y}|\widetilde{\mathbf{y}}|^{B} \partial_{i}\left(\hat{y}_{L}\right) \int_{1}^{\infty} d z \gamma_{l}(z) \mathcal{M}\left(\tau^{i \nu}\right)(\mathbf{y}, t-z|\mathbf{y}| / c) \\
& +\frac{\widetilde{\Delta^{-n}}\left(\hat{x}_{L}\right)}{c^{2}(2 l+3)} \underset{B=0}{\operatorname{FP}} \int d^{3} \mathbf{y}|\widetilde{\mathbf{y}}|^{B} \hat{y}_{L} y_{i} \int_{1}^{\infty} d z \gamma_{l+1}(z) \mathcal{M}\left(\tau^{i \nu}\right)(\mathbf{y}, t-z|\mathbf{y}| / c) \text {. }
\end{aligned}
$$

The sum of these three terms can be transformed so that the function $\mathcal{R}_{L}^{i \nu}$ shows up. Since for any STF tensor $\hat{T}_{L} \partial_{i}\left(\hat{y}_{L}\right)=l \hat{T}_{i L-1} \hat{y}_{L-1}$ and $\hat{T}_{L} \hat{y}_{L} y_{i}=\hat{T}_{L} \hat{y}_{i L}+\frac{l}{2 l+1} \hat{T}_{i L-1} \hat{y}_{L-1}|\mathbf{y}|^{2}$, and keeping in mind that all the multi-indices $L$ will have to be summed, we can write

$$
\begin{aligned}
& \widetilde{\Delta^{-n}}\left(\hat{x}_{L}\right) \underset{B=0}{\mathrm{FP}} \int d^{3} \mathbf{y} \partial_{i}\left(|\widetilde{\mathbf{y}}|^{B}\right) \hat{y}_{L} \int_{1}^{\infty} d z \gamma_{l}(z) \mathcal{M}\left(\tau^{i \nu}\right)(\mathbf{y}, t-z|\mathbf{y}| / c) \\
& +l \widetilde{\Delta^{-n}}\left(\hat{x}_{i L-1}\right) \underset{B=0}{\operatorname{FP}} \int d^{3} \mathbf{y}|\widetilde{\mathbf{y}}|^{B} \hat{y}_{L-1} \int_{1}^{\infty} d z \gamma_{l}(z) \mathcal{M}\left(\tau^{i \nu}\right)(\mathbf{y}, t-z|\mathbf{y}| / c) \\
& +\frac{\widetilde{\Delta^{-n}}\left(\hat{x}_{L}\right)}{c^{2}(2 l+3)} \underset{B=0}{\mathrm{FP}} \int d^{3} \mathbf{y}|\widetilde{\mathbf{y}}|^{B} \hat{y}_{i L} \int_{1}^{\infty} d z \gamma_{l+1}(z) \mathcal{M}\left(\tau^{i \nu}\right)(\mathbf{y}, t-z|\mathbf{y}| / c) \\
& +\frac{l \widetilde{\Delta^{-n}}\left(\hat{x}_{i L-1}\right)}{c^{2}(2 l+1)(2 l+3)} \underset{B=0}{\mathrm{FP}} \int d^{3} \mathbf{y}|\widetilde{\mathbf{y}}|^{B}|\mathbf{y}|^{2} \hat{y}_{L-1} \int_{1}^{\infty} d z \gamma_{l+1}(z) \mathcal{M}\left(\tau^{i \nu}\right)(\mathbf{y}, t-z|\mathbf{y}| / c) .
\end{aligned}
$$

An interesting relation between $\gamma_{l}$-functions : $\frac{d^{2}}{d z^{2}}\left[\gamma_{l+1}(z)\right]=(2 l+1)(2 l+3)\left[\gamma_{l-1}(z)-\gamma_{l}(z)\right]$, after integrating by part the last integral, in terms of $d z$ integration, allows us to get the more explicit form :

$$
\begin{aligned}
\widetilde{\Delta^{-n}}\left(\hat{x}_{L}\right) \partial_{0} \mathcal{R}_{L}^{0 \nu}(t)= & \widetilde{\Delta^{-n}}\left(\hat{x}_{L}\right) \underset{B=0}{\mathrm{FP}} \int d^{3} \mathbf{y} \partial_{i}\left(|\widetilde{\mathbf{y}}|^{B}\right) \hat{y}_{L} \int_{1}^{\infty} d z \gamma_{l}(z) \mathcal{M}\left(\tau^{i \nu}\right)(\mathbf{y}, t-z|\mathbf{y}| / c) \\
& +l \widetilde{\Delta^{-n}}\left(\hat{x}_{i L-1}\right) \mathcal{R}_{L-1}^{i \nu}(t)+\frac{\widetilde{\Delta^{-n}}\left(\hat{x}_{L}\right)}{c^{2}(2 l+3)} \mathcal{R}_{i L}^{(2)}(t) .
\end{aligned}
$$

Summing up Eqs. (5.12) and (5.17) we obtain

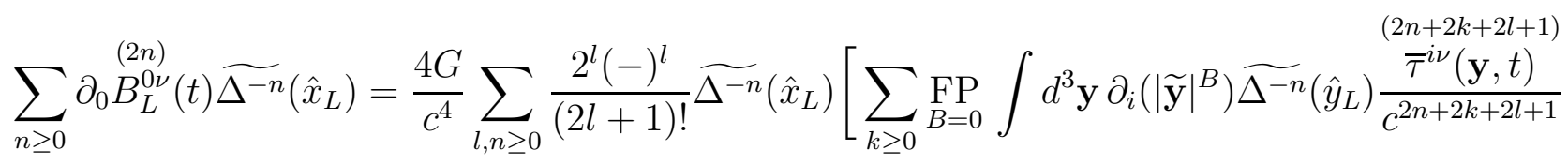

$$
\begin{aligned}
& \left.+\underset{B=0}{\mathrm{FP}} \int d^{3} \mathbf{y} \partial_{i}\left(|\widetilde{\mathbf{y}}|^{B}\right) \hat{y}_{L} \int_{1}^{\infty} d z \gamma_{l}(z) \frac{(2 n+2 l+1)}{\left.c^{2 n+2 l+1}\right)}(\mathbf{y}, t-z|\mathbf{y}| / c)\right]
\end{aligned}
$$

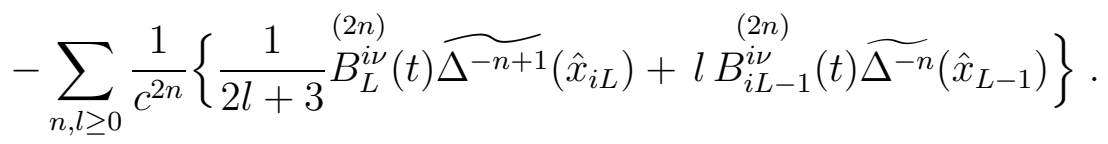


The last line cancels out the terms coming from Eq. (5.8).

We can therefore write down the result for the divergence of $\bar{h}^{\mu \nu}$ which, at this stage, depends only on terms with integrals of $\partial_{i}\left(|\widetilde{\mathbf{y}}|^{B}\right)$ and having the spatial structure given by $\widetilde{\Delta^{-n}}\left(\hat{x}_{L}\right)$. After summing Eqs. (5.6), (5.8) and (5.18) we get

$$
\begin{aligned}
& \partial_{\mu} \bar{h}^{\mu \nu}= \\
& -\frac{4 G}{c^{4}} \sum_{n, l, k \geq 0} \frac{(-)^{l}}{l !} \frac{\widetilde{\Delta^{-n}}\left(\hat{x}_{L}\right)}{c^{2 k+2 n}} \underset{B=0}{\mathrm{FP}} \int_{|\mathbf{y}|>\mathcal{R}} d^{3} \mathbf{y} \partial_{i}\left(|\widetilde{\mathbf{y}}|^{B}\right) \widetilde{\Delta^{-k}}\left(\hat{\partial}_{L}\left(|\mathbf{y}|^{-1}\right)\right) \stackrel{(2 n+2 k)}{\mathcal{M}}\left(\bar{\tau}^{i \nu}\right)(\mathbf{y}, t) \\
& +\frac{4 G}{c^{4}} \sum_{n, l, k \geq 0} \frac{2^{l}(-)^{l}}{(2 l+1) !} \frac{\widetilde{\Delta^{-n}}\left(\hat{x}_{L}\right)}{c^{2 n+2 k+2 l+1}} \underset{B=0}{\mathrm{FP}} \int_{|\mathbf{y}|>\mathcal{R}} d^{3} \mathbf{y} \partial_{i}\left(|\widetilde{\mathbf{y}}|^{B}\right) \widetilde{\Delta^{-k}}\left(\hat{y}_{L}\right) \mathcal{M}\left(\bar{\tau}^{i \nu}\right)(\mathbf{y}, t)
\end{aligned}
$$

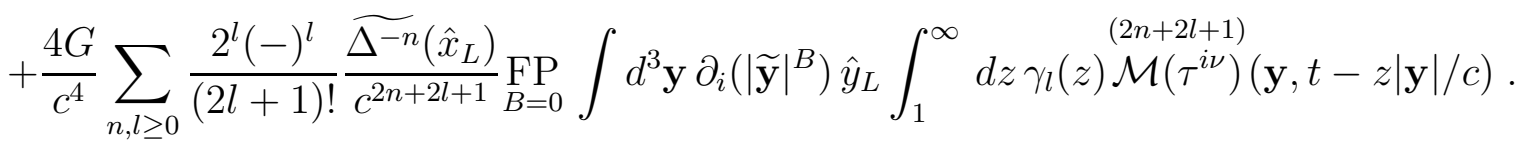

In the second term we have used the fact that the integral depends only on the values for which $|\mathbf{y}|>\mathcal{R}$ to write $\bar{\tau}^{i \nu}=\mathcal{M}\left(\bar{\tau}^{i \nu}\right)$ on that domain. The last term of Eq. (5.19) depends on a retarded integral of the multipolar post-Minkowskian expansion $\mathcal{M}\left(\tau^{i \nu}\right)$. By integrating by part the integral over $z$ one can transform this last term into

$$
\begin{aligned}
\frac{4 G}{c^{4}} \sum_{l, n \geq 0} & \frac{2^{l}(-)^{l}}{(2 l+1) !} \widetilde{\Delta^{-n}}\left(\hat{x}_{L}\right) \\
& \times \sum_{p \geq 0} \frac{1}{c^{2 n-p+l}} \underset{B=0}{\mathrm{FP}} \int_{|\mathbf{y}|<\mathcal{R}} d^{3} \mathbf{y} \partial_{i}\left(|\widetilde{\mathbf{y}}|^{B}\right) \hat{y}_{L}|\mathbf{y}|^{-p-l-1} \stackrel{(p+l)}{\gamma_{l}}(1) \stackrel{(2 n-p+l)}{\mathcal{M}\left(\tau^{i \nu}\right)(\mathbf{y}, t-|\mathbf{y}| / c) .}
\end{aligned}
$$

The superscript $(p+l)$ on the $\gamma_{l}$-function refers to the $z$-differentiation. It is straightforward to show, using the fact that $\stackrel{(l)}{\gamma}_{l}(z)=(-)^{l+1}(2 l+1) ! ! P_{l}(z)$ is directly related to the Legendre polynomial, that

$$
\stackrel{(p+l)}{\gamma_{l}}(1)=(-)^{l+1} \frac{(2 l+1) ! !(l+p) !}{2^{p} p !(l-p) !} .
$$

Since $\mathcal{M}\left(\tau^{i \nu}\right)$ is singular at the origin (but regular at infinity), and because of the explicit factor $B$ brought about by the derivative $\partial_{i}\left(|\widetilde{\mathbf{y}}|^{B}\right)$, the integral in Eq. (5.20) ranges over $|\mathbf{y}|<\mathcal{R}$ (and even $|\mathbf{y}|<\epsilon$ where $\epsilon$ is an arbitrary small number). We can then expand $\mathcal{M}\left(\tau^{i \nu}\right)(\mathbf{y}, t-|\mathbf{y}| / c)$ when $c \rightarrow+\infty$. Furthermore we can change the integration over 
$|\mathbf{y}|<\mathcal{R}$ into an integration over $|\mathbf{y}|>\mathcal{R}$ by simply changing the sign in front of the integral. Indeed, this comes from a technical lemma, which plays an important role in Refs. [39.40]; see before Eq. (C6) in Appendix Q, and the proof given in Ref. [51]. Thus,

$$
\begin{aligned}
& -\frac{4 G}{c^{4}} \sum_{n, l, k \geq 0} \frac{2^{l}(-)^{l}}{(2 l+1) !} \widetilde{\Delta^{-n}}\left(\hat{x}_{L}\right) \\
& \quad \times \sum_{p \geq 0} \frac{1}{c^{2 n-p+l+k}} \mathrm{FP}_{B=0} \int_{|\mathbf{y}|>\mathcal{R}} d^{3} \mathbf{y} \partial_{i}\left(|\widetilde{\mathbf{y}}|^{B}\right) \hat{n}_{L} \frac{(-)^{k}}{k !}|\mathbf{y}|^{k-p-1} \stackrel{(p+l)}{\gamma_{l}}(1) \mathcal{M}\left(\tau^{i \nu}\right)(\mathbf{y}, t) .
\end{aligned}
$$

By changing the label $k$ into $2 k+p-l$ and $2 k+1+p-l$, in order to cover odd and even numbers, we are able to write the previous expression in terms of some sums of real numbers indexed by $p$, i.e.

$$
\begin{aligned}
-\frac{4 G}{c^{4}} \sum_{n, l, k \geq 0} & \frac{2^{l}(-)^{l}}{(2 l+1) !} \widetilde{\Delta^{-n}}\left(\hat{x}_{L}\right)\left\{\sum_{p=0}^{l} \frac{(-1)^{p+l} \gamma_{l}^{(p+l)}(1)}{(p-l+2 k) !}\right\} \\
& \times \underset{B=0}{\mathrm{FP}} \int_{|\mathbf{y}|>\mathcal{R}} d^{3} \mathbf{y} \partial_{i}\left(|\widetilde{\mathbf{y}}|^{B}\right) \hat{n}_{L}|\mathbf{y}|^{2 k-l-1} \mathcal{M}\left(\tau^{i \nu}\right)(\mathbf{y}, t) \\
+\frac{4 G}{c^{4}} \sum_{n, l, k \geq 0} & \frac{2^{l}(-)^{l}}{(2 l+1) !} \widetilde{\Delta^{-n}}\left(\hat{x}_{L}\right)\left\{\sum_{p=0}^{l} \frac{(-1)^{p+l}{ }^{(p+l)}(1)}{(p-l+2 k+1) !}\right\} \\
& \times \underset{B=0}{\mathrm{FP}} \int_{|\mathbf{y}|>\mathcal{R}} d^{3} \mathbf{y} \partial_{i}\left(|\widetilde{\mathbf{y}}|^{B}\right) \hat{n}_{L}|\mathbf{y}|^{2 k-l} \mathcal{M}\left(\tau^{i \nu}\right)(\mathbf{y}, t) .
\end{aligned}
$$

The sums in curly brackets are found to be explicit expressions depending on $k$ and $l$ and some factorial combinations :

$$
\begin{aligned}
& \forall k \geq l+1, \quad \sum_{p=0}^{l} \frac{(-1)^{p+l} \gamma_{l}^{(p+l)}(1)}{(p-l+2 k) !}=-\frac{(2 l+1) ! !}{2^{k} k !(2 k-2 l-1) ! !}, \\
& \forall k \geq l, \quad \sum_{p=0}^{l} \frac{(-1)^{p+l} \gamma_{l}^{(p+l)}(1)}{(p-l+2 k+1) !}=-\frac{(2 l+1) ! !}{2^{k-l}(k-l) !(2 k+1) ! !}, \\
& \forall k \leq l, \quad \sum_{p=0}^{l} \frac{(-1)^{p+l} \gamma_{l}^{(p+l)}(1)}{(p-l+2 k) !}=(-1)^{k+l+1} \frac{(2 l+1) ! !(2 l-2 k-1) ! !}{2^{k} k !}, \\
& \forall k \leq l-1, \quad \sum_{p=0}^{l} \frac{(-1)^{p+l} \gamma_{l}^{(p+l)}(1)}{(p-l+2 k+1) !}=0 .
\end{aligned}
$$

Thanks to these formulas, one can transform Eq. (5.23) into 


$$
\begin{aligned}
& \frac{4 G}{c^{4}} \sum_{n, l \geq 0} \sum_{k \geq l+1} \frac{(-)^{l}}{l !} \frac{1}{2^{k} k !(2 k-2 l-1) ! !} \widetilde{\Delta^{-n}}\left(\hat{x}_{L}\right) \\
& \times \underset{B=0}{\operatorname{FP}} \int_{|\mathbf{y}|>\mathcal{R}} d^{3} \mathbf{y} \partial_{i}\left(|\widetilde{\mathbf{y}}|^{B}\right) \hat{n}_{L}|\mathbf{y}|^{2 k-l-1} \mathcal{M}\left(\tau^{i \nu}\right)(\mathbf{y}, t) \\
&+\frac{4 G}{c^{4}} \sum_{n, l \geq 0} \sum_{k \leq l} \frac{(-)^{k}(2 l-2 k-1) ! !}{l ! 2^{k} k !} \widetilde{\Delta^{-n}}\left(\hat{x}_{L}\right) \\
& \quad \times \underset{B=0}{\operatorname{FP}} \int_{|\mathbf{y}|>\mathcal{R}} d^{3} \mathbf{y} \partial_{i}\left(|\widetilde{\mathbf{y}}|^{B}\right) \hat{n}_{L}|\mathbf{y}|^{2 k-l-1} \mathcal{M}\left(\tau^{i \nu}\right)(\mathbf{y}, t) \\
&-\frac{4 G}{c^{4}} \sum_{n, l \geq 0} \sum_{k \geq l} \frac{(-)^{l}}{l !} \frac{1}{2^{k-l}(2 k+1) ! !(k-l) !} \widetilde{\Delta^{-n}}\left(\hat{x}_{L}\right) \\
& \quad \times \underset{B=0}{\mathrm{FP}} \int_{|\mathbf{y}|>\mathcal{R}} d^{3} \mathbf{y} \partial_{i}\left(|\widetilde{\mathbf{y}}|^{B}\right) \hat{n}_{L}|\mathbf{y}|^{2 k-l} \mathcal{M}\left(\tau^{i \nu}\right)(\mathbf{y}, t) .
\end{aligned}
$$

In the latter expression we can recognize

$$
\begin{aligned}
\forall k \geq l, \quad \widetilde{\Delta^{-k}}\left(\hat{\partial}_{L}\left(|\mathbf{y}|^{-1}\right)\right) & =\frac{1}{(2 k-2 l-1) ! ! 2^{k} k !} \hat{n}_{L}|\mathbf{y}|^{2 k-l-1}, \\
\forall k \leq l, \quad \widetilde{\Delta^{-k}}\left(\hat{\partial}_{L}\left(|\mathbf{y}|^{-1}\right)\right) & =\frac{(-)^{k+l}(2 l-2 k-1) ! !}{2^{k} k !} \hat{n}_{L}|\mathbf{y}|^{2 k-l-1}, \\
\widetilde{\Delta^{-k}}\left(\hat{y}_{L}\right) & =\frac{(2 l+1) ! !}{2^{k} k !(2 k+2 l+1) ! !} \hat{y}_{L}|\mathbf{y}|^{2 k}
\end{aligned}
$$

so that we obtain

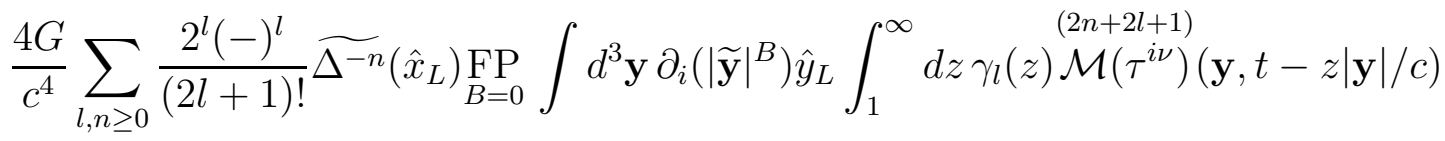

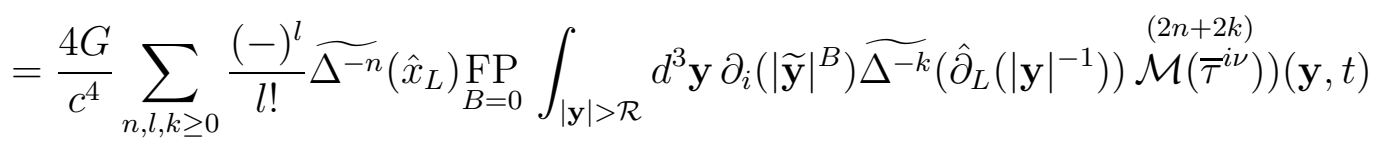

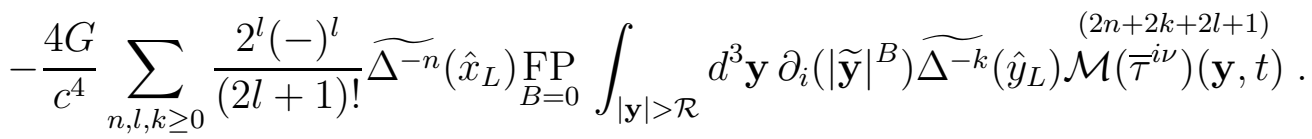

After replacing Eq. (5.32) in Eq. (5.19), at long last we find

$$
\partial_{\mu} \bar{h}^{\mu \nu}=0
$$

In this way, we have checked that the post-Newtonian metric, found by matching as a definite functional of the stress-energy pseudo-tensor $\tau^{\mu \nu}$, satisfies the harmonic-coordinate condition as a consequence of the conservation of this pseudo-tensor. 


\section{APPENDIX A: NEAR-ZONE EXPANSION OF THE RETARDED INTEGRAL}

This appendix, provided here for completeness, is an extended, and also somewhat simplified, version of the derivation given in Appendix A of Ref. [32. We are interested in source functions, say $\mathcal{M}(\tau)(\mathbf{x}, t)$, having the form of an exterior multipole-moment decomposition, valid outside the compact-support domain of the source. We employ the same notation as in Section IIA (except that we do not write the space-time indices) : $\tau$ denotes

the pseudo-tensor of the source; notably we have $\mathcal{M}(\tau)=\frac{c^{4}}{16 \pi G} \mathcal{M}(\Lambda)$ where $\Lambda$ is the gravitational source term. The two basic properties of the function $\mathcal{M}(\tau)(\mathbf{x}, t)$ are that it is smooth on $\mathbb{R}^{4}$ deprived from the spatial origin $r=0$ :

$$
\mathcal{M}(\tau)(\mathbf{x}, t) \in C^{\infty}\left(\mathbb{R}_{*}^{3} \times \mathbb{R}\right)
$$

and that it admits a near-zone expansion, when $r \rightarrow 0$ (with $t=$ const), having the appropriate structure $[c f$ Eq. (2.14)] : i.e., $\forall N \in \mathbb{N}$,

$$
\mathcal{M}(\tau)(\mathbf{x}, t)=\sum \hat{n}_{L} r^{a}(\ln r)^{p} G_{L, a, p}(t)+\mathcal{O}\left(r^{N}\right)
$$

where $a \in \mathbb{Z}$ with $a \leq N$ and $p \in \mathbb{N}$. Like in Section IIB we denote with an overline the formal (infinite) near-zone expansion,

$$
\overline{\mathcal{M}(\tau)(\mathbf{x}, t)}=\sum \hat{n}_{L} r^{a}(\ln r)^{p} G_{L, a, p}(t)
$$

It is very important to make the distinction between $\mathcal{M}(\tau)$ and its formal near-zone expansion $\overline{\mathcal{M}(\tau)}$. Here we shall investigate the retarded integral of the product $r^{B} \mathcal{M}(\tau)(\mathbf{x}, t)$, where $B \in \mathbb{C}$, by means of analytic continuation (we pose $r_{0}=1$ in this Appendix). For this task we assume at first that the real part of $B$ is large enough so as to "kill" the divergencies, when $r \rightarrow 0$, of the expansion (A2), so that the retarded integral is initially well-defined. Therefore, rigorously speaking, we are allowed to do this only if there exists a finite maximal 
divergency, i.e. some $a_{\text {min }} \leq a$ in Eq. (A2) with finite $a_{\text {min }} \in \mathbb{Z}$. We have seen in Section IIA that such maximal divergency exists at any given post-Minkowskian order $m$, but no longer exists for the full post-Minkowskian series because $a_{\min }(m) \rightarrow-\infty$ when $m \rightarrow+\infty$. The consequence is that the analytic continuation is in principle justified only at a given finite post-Minkowskian order. But, as explained in Section П1 A, we sum up systematically all the post-Minkowskian results. In this way we are entitled to proceed as we do below; simply we have to remember that the end result will be a priori true only in a sense of formal post-Minkowskian expansions.

We decompose the source term into multipoles according to

$$
\mathcal{M}(\tau)(\mathbf{x}, t)=\sum_{l=0}^{+\infty} \hat{n}_{L} \sigma_{L}(r, t)
$$

where the $\sigma_{L}$ 's are STF functions in $L=i_{1} \cdots i_{l}$. The inverse formula is

$$
\sigma_{L}(r, t)=\frac{(2 l+1) ! !}{l !} \int \frac{d \Omega}{4 \pi} \hat{n}_{L} \mathcal{M}(\tau)(\mathbf{x}, t)
$$

where $d \Omega$ is the solid-angle element around the unit vector $n_{i}=n^{i}=x^{i} / r$. Then the expression of the retarded integral, in a sense of analytic continuation in $B$, is given by the following explicit formula, obtained in Ref. [36] [see Eqs. (6.3)-(6.5) there] :

$$
\square_{\text {Ret }}^{-1}\left[r^{B} \mathcal{M}(\tau)(\mathbf{x}, t)\right]=\sum_{l=0}^{+\infty} \int_{-\infty}^{t-r} d s \hat{\partial}_{L}\left\{\frac{R_{L}^{B}\left(\frac{t-r-s}{2}, s\right)-R_{L}^{B}\left(\frac{t+r-s}{2}, s\right)}{r}\right\}
$$

(we pose $c=1$ and $r_{0}=1$ in this Appendix), where the function $R_{L}^{B}(\rho, s)$ reads

$$
R_{L}^{B}(\rho, s)=\rho^{l} \int_{0}^{\rho} d x \frac{(\rho-x)^{l}}{l !}\left(\frac{2}{x}\right)^{l-1} x^{B} \sigma_{L}(x, x+s)
$$

Following the same procedure as in Eqs. (A6)-(A7) in Ref. [32] we are allowed to re-write the expression (A6) into the alternative form 


$$
\begin{aligned}
\square_{\text {Ret }}^{-1}\left[r^{B} \mathcal{M}(\tau)(\mathbf{x}, t)\right] & =\sum_{l=0}^{+\infty} \int_{-r}^{r} d u \hat{\partial}_{L}\left\{\frac{1}{r} R_{L}^{B}\left(\frac{u+r}{2}, t-u\right)\right\} \\
& -\frac{1}{4 \pi} \sum_{l=0}^{+\infty} \frac{(-)^{l}}{l !} \hat{\partial}_{L}\left[\frac{\mathcal{R}_{L}^{B}(t-r)-\mathcal{R}_{L}^{B}(t+r)}{2 r}\right] .
\end{aligned}
$$

The "anti-symmetric" wave is parametrized by $\mathcal{R}_{L}^{B}(t)$ which is related to the function $R_{L}^{B}(\rho, s)$ by

$$
\mathcal{R}_{L}^{B}(t)=8 \pi(-)^{l+1} l ! \int_{-\infty}^{t} d s R_{L}^{B}\left(\frac{t-s}{2}, s\right) .
$$

Inserting Eq. (A7), and performing some change of variables, we obtain

$$
\mathcal{R}_{L}^{B}(t)=\frac{4 \pi l !}{(2 l+1) ! !} \int_{0}^{+\infty} d x x^{B+l+2} \int_{1}^{+\infty} d z \gamma_{l}(z) \sigma_{L}(x, t-z x)
$$

and, using the relation (A5), and considering the variable $x$ as the norm of $\mathbf{x} \in \mathbb{R}^{3}$, we further get

$$
\mathcal{R}_{L}^{B}(t)=\int d^{3} \mathbf{x}|\mathbf{x}|^{B} \hat{x}_{L} \int_{1}^{+\infty} d z \gamma_{l}(z) \mathcal{M}(\tau)(\mathbf{x}, t-z|\mathbf{x}|)
$$

In these expressions the function $\gamma_{l}(z)$ is defined by

$$
\gamma_{l}(z)=(-)^{l+1} \frac{(2 l+1) ! !}{2^{l} l !}\left(z^{2}-1\right)^{l}
$$

where the particular $l$-dependent factor has been chosen in such a way that the integral is normalized to one in the following sense (see Ref. [32]). Considering first that $l$ is a complex number such that $-1<\Re(l)<-1 / 2$ we can compute the integral of $\gamma_{l}(z)$ by means of the Euler $\Gamma$-function, with the result

$$
\int_{1}^{+\infty} d z \gamma_{l}(z)=2(-)^{l+1} \frac{\Gamma(2 l+2) \Gamma(-2 l-1)}{\Gamma(l+1) \Gamma(-l)} .
$$


The right-hand-side of this equation can be analytically continued to all values $l \in \mathbb{C}$ except half-integer values, and is found to be equal to one when $l$ is an integer :

$$
\int_{1}^{+\infty} d z \gamma_{l}(z)=1 \quad(l \in \mathbb{N})
$$

Next, let us treat the first term in the right-hand-side of Eq. (A8), say

$$
J^{B}(\mathbf{x}, t) \equiv \sum_{l=0}^{+\infty} \int_{-r}^{r} d u \hat{\partial}_{L}\left\{\frac{1}{r} R_{L}^{B}\left(\frac{u+r}{2}, t-u\right)\right\}
$$

This term is a particular solution of the d'Alembertian equation $\square J^{B}=r^{B} \mathcal{M}(\tau)$ [since the second term in Eq. (A8) is a source-free solution]. We shall prove that the (formal) nearzone expansion of that term, i.e. $\overline{J^{B}(\mathbf{x}, t)}$, is given by the integral of the "instantaneous" potentials acting on the near-zone expansion of the source term, i.e. $r^{B} \overline{\mathcal{M}(\tau)(\mathbf{x}, t)}$. For any of the terms composing the multipolar source $r^{B} \overline{\mathcal{M}(\tau)}$ [see Eq. (A3)], we first define

$$
\Delta^{-1}\left[\hat{n}_{L} r^{B+a}(\ln r)^{p} G_{L, a, p}(t)\right]=\left(\frac{d}{d B}\right)^{p}\left[\frac{\hat{n}_{L} r^{B+a+2} G_{L, a, p}(t)}{(B+a+2-l)(B+a+3+l)}\right]
$$

(this being justified by the fact that one gets an identity by applying $\Delta$ on both sides). Clearly the previous formula can be iterated and so we can define the operator $\Delta^{-k-1} \equiv$ $\left(\Delta^{-1}\right)^{k+1}$, applied on each separate terms in Eq. (A3) and therefore on the complete series $r^{B} \overline{\mathcal{M}(\tau)(\mathbf{x}, t)}$. From this we obtain the instantaneous-potentials operator, as the formal expansion series

$$
\mathcal{I}^{-1}\left[r^{B} \overline{\mathcal{M}(\tau)(\mathbf{x}, t)}\right]=\sum_{k=0}^{+\infty}\left(\frac{\partial}{c \partial t}\right)^{2 k} \Delta^{-k-1}\left[r^{B} \overline{\mathcal{M}(\tau)(\mathbf{x}, t)}\right]
$$

Notice that this operator $\mathcal{I}^{-1}$ contains only some even powers of $1 / c$. An important point for our purpose is that $\mathcal{I}^{-1}\left[r^{B} \overline{\mathcal{M}(\tau)}\right]$ is proportional to the regularization factor $r^{B}$; and it evidently satisfies $\square\left(\mathcal{I}^{-1}\left[r^{B} \overline{\mathcal{M}(\tau)}\right]\right)=r^{B} \overline{\mathcal{M}(\tau)}$. On the other hand, we have also the 
equation $\square \overline{J^{B}}=r^{B} \overline{\mathcal{M}(\tau)}$, which comes from applying the overline operation onto $\square J^{B}=$ $r^{B} \mathcal{M}(\tau)$. This shows that $r^{B} \overline{\mathcal{M}(\tau)}$ and $\overline{J^{B}}$ must differ by a solution of the homogeneous equation, hence there should exist some functions $C_{L}^{B}(t)$ and $D_{L}^{B}(t)$ such that

$$
\overline{J^{B}(\mathbf{x}, t)}=\mathcal{I}^{-1}\left[r^{B} \overline{\mathcal{M}(\tau)(\mathbf{x}, t)}\right]+\sum_{l=0}^{+\infty} \hat{\partial}_{L}\left\{\frac{\overline{C_{L}^{B}(t-r)+D_{L}^{B}(t+r)}}{r}\right\} .
$$

Note that the dependence on $B$ of the second term is "hidden" inside the functions $C_{L}^{B}$ and $D_{L}^{B}$. Let us now prove that in fact the latter functions must be zero. This is a simple consequence of the expression (A7) for the function $R_{L}^{B}(\rho, s)$, from which we deduce that the expansion when $\rho \rightarrow 0$ of this function is proportional to $\rho^{B}$; in fact, it has the structure $R_{L}^{B}(\rho, s) \sim \sum \rho^{B+b}(\ln \rho)^{q}$, when $\rho \rightarrow 0$. From this knowledge, we easily find that the nearzone expansion of $\overline{J^{B}}$ is proportional to the factor $r^{B}$. Since, as we have remarked, this is also the case of the first term in Eq. (A18), $\mathcal{I}^{-1}\left[r^{B} \overline{\mathcal{M}(\tau)}\right]$, and since it is impossible that (the near-zone expansion of) the second term in Eq. (A18) be itself proportional to $r^{B}$ - the $B$ 's affect only the functions $C_{L}^{B}$ and $D_{L}^{B}$ but not the structure of the near-zone expansion - we conclude that $C_{L}^{B}$ and $D_{L}^{B}$ are identically zero. Hence we have proved

$$
\overline{J^{B}(\mathbf{x}, t)}=\mathcal{I}^{-1}\left[r^{B} \overline{\mathcal{M}(\tau)(\mathbf{x}, t)}\right] .
$$

It suffices now to apply the overline operation (i.e., to take the near-zone expansion) onto Eq. (A8) to get our final result,

$$
\overline{\square_{\text {Ret }}^{-1}\left[r^{B} \mathcal{M}(\tau)(\mathbf{x}, t)\right]}=\mathcal{I}^{-1}\left[r^{B} \overline{\mathcal{M}(\tau)(\mathbf{x}, t)}\right]-\frac{1}{4 \pi} \sum_{l=0}^{+\infty} \frac{(-)^{l}}{l !} \hat{\partial}_{L}\left[\frac{\overline{\mathcal{R}_{L}^{B}(t-r)-\mathcal{R}_{L}^{B}(t+r)}}{2 r}\right]
$$

where we recall that the function $\mathcal{R}_{L}^{B}(t)$ has been given by Eq. (A11). [The formula used Section $\llbracket \mathrm{IB}$ results from applying the finite part operation $\underset{B=0}{\mathrm{FP}}$.] 


\section{APPENDIX B: THE GENERALIZED POISSON OPERATOR}

In Appendix $\mathrm{A}$ we have been interested in source functions of the multipolar type $\mathcal{M}(\tau)(\mathbf{x}, t)$, which are smooth in $\mathbb{R}_{*}^{3} \times \mathbb{R}$ and possess a near-zone expansion of the type (A3). In the present Appendix B we consider some source functions of the post-Newtonian type $\bar{\tau}(\mathbf{x}, t)$. These are supposed to be smooth all over $\mathbb{R}^{4}$,

$$
\bar{\tau}(\mathbf{x}, t) \in C^{\infty}\left(\mathbb{R}^{4}\right),
$$

and to admit a far-zone expansion with structure $(\forall N \in \mathbb{N})$

$$
\bar{\tau}(\mathbf{x}, t)=\sum \hat{n}_{L} r^{a}(\ln r)^{p} G_{L, a, p}(t)+S_{N}(\mathbf{x}, t)
$$

where $a \in \mathbb{Z}$, with $-N \leq a$, and $p \in \mathbb{N}$. The remainder-term is $S_{N}(\mathbf{x}, t)=\mathcal{O}\left(1 / r^{N}\right)$ when $r \rightarrow+\infty$ with $t=$ const.

Let us consider some $B \in \mathbb{C}$, and a radius $\mathcal{R} \in \mathbb{R}$ with $\mathcal{R}>0$. We define two integrals, corresponding to a split of the Poisson integral between "near-zone" and "far-zone" contributions, separated by the radius $\mathcal{R}$ :

$$
\begin{aligned}
& I_{<}^{B}(\mathbf{x}, t)=-\frac{1}{4 \pi} \int_{|\mathbf{y}|<\mathcal{R}} \frac{d^{3} \mathbf{y}}{|\mathbf{x}-\mathbf{y}|}|\widetilde{\mathbf{y}}|^{B} \bar{\tau}(\mathbf{y}, t), \\
& I_{>}^{B}(\mathbf{x}, t)=-\frac{1}{4 \pi} \int_{|\mathbf{y}|>\mathcal{R}} \frac{d^{3} \mathbf{y}}{|\mathbf{x}-\mathbf{y}|}|\widetilde{\mathbf{y}}|^{B} \bar{\tau}(\mathbf{y}, t) .
\end{aligned}
$$

The $B$-dependent regularization factor is $|\widetilde{\mathbf{y}}|^{B} \equiv\left(|\mathbf{y}| / r_{0}\right)^{B}$. It is easily checked that the nearzone integral $I_{<}^{B}(\mathbf{x}, t)$ is well-defined (convergent) when $\Re(B)>-3$ and that the far-zone one $I_{>}^{B}(\mathbf{x}, t)$ is well-defined when $\Re(B)<-a_{\max }-2$, where $a_{\max }$ is the maximal power of $r$ in the expansion (B2). So we have to assume at this stage the existence of some maximal divergency corresponding to some power $a_{\max }$. Strictly speaking, our present investigation is thus valid only at some finite post-Newtonian order. But, in fine, we sum up the results, and we consider the complete post-Newtonian series to hold true in a formal sense. 
We want first to check that the integrals (B3) and (B4) can be analytically continued down to a neighbourhood of $B=0$ (except at the value $B=0$ itself), let say in the open domain $\mathcal{B}_{\epsilon}$ defined by $0<|B|<\epsilon$ (where $\epsilon<1$ ). There is no problem with the nearzone integral $I_{<}^{B}(\mathbf{x}, t)$ which is clearly convergent all over $\mathcal{B}_{\epsilon}$ and even at the value $B=0$. Concerning the far-zone integral $I_{>}^{B}(\mathbf{x}, t)$ we replace the function $\bar{\tau}$ inside the integrand by its far-zone expansion $(\overline{\mathrm{B} 2})$ :

$$
I_{>}^{B}(\mathbf{x}, t)=-\frac{1}{4 \pi} \int_{|\mathbf{y}|>\mathcal{R}} \frac{d^{3} \mathbf{y}|\widetilde{\mathbf{y}}|^{B}}{|\mathbf{x}-\mathbf{y}|}\left\{\sum \hat{n}_{L}(\mathbf{y})|\mathbf{y}|^{a}(\ln |\mathbf{y}|)^{p} G_{L, a, p}(t)+S_{N}(\mathbf{y}, t)\right\} .
$$

When $N$ is large enough the contribution due to the remainder $S_{N}$ is convergent all over $\mathcal{B}_{\epsilon}$ and at $B=0$, with evidently the value at $B=0$ given by

$$
\int_{|\mathbf{y}|>\mathcal{R}} \frac{d^{3} \mathbf{y}|\widetilde{\mathbf{y}}|^{B}}{|\mathbf{x}-\mathbf{y}|} S_{N}(\mathbf{y}, t)=\int_{|\mathbf{y}|>\mathcal{R}} \frac{d^{3} \mathbf{y}}{|\mathbf{x}-\mathbf{y}|} S_{N}(\mathbf{y}, t)+\mathcal{O}(B) .
$$

Thus we need only to deal with the other contributions, which consist of a finite sum of terms, say

$$
\sum \int_{|\mathbf{y}|>\mathcal{R}} \frac{d^{3} \mathbf{y}|\widetilde{\mathbf{y}}|^{B}}{|\mathbf{x}-\mathbf{y}|} \hat{n}_{L}(\mathbf{y})|\mathbf{y}|^{a}(\ln |\mathbf{y}|)^{p}
$$

Let us suppose that the field point $\mathbf{x}$ lies inside the far-zone domain, i.e. $\mathcal{R}<|\mathbf{x}|$. We distinguish the two cases where $|\mathbf{y}|<|\mathbf{x}|$ and $|\mathbf{x}|<|\mathbf{y}|$. For each of these two cases we substitute into the integrals the appropriate multipolar expansion of the factor $\frac{1}{|\mathbf{x}-\mathbf{y}|}$, for instance $\frac{1}{|\mathbf{x}-\mathbf{y}|}=\sum_{l=0}^{+\infty} \frac{(-)^{l}}{l !} y^{L} \hat{\partial}_{L} \frac{1}{|\mathbf{x}|}$ when $|\mathbf{y}|<|\mathbf{x}|$. This leads, after performing the integration over the angles, to some series of radial integrals having the structure (ignoring some unimportant factors)

$$
\sum \frac{\hat{x}_{L}}{|\mathbf{x}|^{2 l+1}} \int_{\mathcal{R}}^{|\mathbf{x}|} d|\mathbf{y}||\mathbf{y}|^{B+a+l+2}(\ln |\mathbf{y}|)^{p}+\sum \hat{x}_{L} \int_{|\mathbf{x}|}^{+\infty} d|\mathbf{y}||\mathbf{y}|^{B+a-l+1}(\ln |\mathbf{y}|)^{p}
$$


When $|\mathbf{x}|<\mathcal{R}$ the reasoning is the same but simply one ignores the first term in Eq. (B8) and take $\mathcal{R}$ as lower bound in the second term. Computing each of these integrals we find

$$
\sum \frac{\hat{x}_{L}}{|\mathbf{x}|^{2 l+1}}\left(\frac{d}{d B}\right)^{p}\left[\frac{|\mathbf{x}|^{B+a+l+3}-\mathcal{R}^{B+a+l+3}}{B+a+l+3}\right]+\sum \hat{x}_{L}\left(\frac{d}{d B}\right)^{p}\left[\frac{-|\mathbf{x}|^{B+a-l+2}}{B+a-l+2}\right] .
$$

Each of these terms clearly admits an analytic continuation for any $B \in \mathcal{B}_{\epsilon}$ and in fact for any $B \in \mathbb{C}$ except at integer values. Furthermore we see from that expression that the function will admit a Laurent expansion when $B \rightarrow 0$, with in general some multiple poles [coming from the differentiation $(d / d B)^{p}$ of simple poles $\left.\sim 1 / B\right]$. Hence our statement.

It is clear that the Laplacians of the two integrals $I_{<}^{B}$ and $I_{>}^{B}$ satisfy, in the domains of the complex plane where these functions were initially valid :

$$
\begin{aligned}
\Re(B)>-3 & \Longrightarrow \Delta I_{<}^{B}(\mathbf{x}, t)=Y(\mathcal{R}-|\mathbf{x}|)|\widetilde{\mathbf{x}}|^{B} \bar{\tau}(\mathbf{x}, t), \\
\Re(B)<-a_{\max }-2 & \Longrightarrow \Delta I_{>}^{B}(\mathbf{x}, t)=Y(|\mathbf{x}|-\mathcal{R})|\widetilde{\mathbf{x}}|^{B} \bar{\tau}(\mathbf{x}, t),
\end{aligned}
$$

where $Y$ denotes the Heaviside step-function. Therefore, if we define for any $B \in \mathcal{B}_{\epsilon}$ the object

$$
I^{B}(\mathbf{x}, t)=I_{<}^{B}(\mathbf{x}, t)+\text { analytic } \underset{B \in \mathcal{B}_{\epsilon}}{\text { continuation }}\left\{I_{>}^{B}(\mathbf{x}, t)\right\}
$$

we find that it necessarily satisfies, for any $B \in \mathcal{B}_{\epsilon}$, the $B$-dependent Poisson equation

$$
\Delta I^{B}(\mathbf{x}, t)=|\widetilde{\mathbf{x}}|^{B} \bar{\tau}(\mathbf{x}, t)
$$

On the other hand, we have learned from Eq. (B9) that $I^{B}$ admits when $B \rightarrow 0$ a Laurent expansion involving (in general) simple and multiple poles. Now the key idea, as we shall prove, is that the finite part, or coefficient of the zero-th power of $B$ in the latter Laurent expansion, represents a particular solution of the Poisson equation that we want to solve. Let the Laurent expansion of $I^{B}$ be 


$$
I^{B}(\mathbf{x}, t)=\sum_{k=k_{\min }}^{+\infty} i_{k}(\mathbf{x}, t) B^{k}
$$

where $k_{\min } \in \mathbb{Z}$, and where the coefficients $i_{k}$ depend on the field point $(\mathbf{x}, t)$. By applying the Laplacian operator onto both sides of Eq. (B14), and using the result (B13) together with the Taylor expansion of the regularization factor $|\widetilde{\mathbf{x}}|^{B}$, we arrive at

$$
\begin{aligned}
k_{\min } \leq k \leq-1 & \Longrightarrow \Delta i_{k}=0 \\
k \geq 0 & \Longrightarrow \Delta i_{k}=\frac{(\ln |\widetilde{\mathbf{x}}|)^{k}}{k !} \bar{\tau} .
\end{aligned}
$$

Thus, the case $k=0$ shows that the finite-part coefficient in the expansion (B14), namely $i_{0}$, is a particular solution of the required equation : $\Delta i_{0}=\bar{\tau}$. We shall now forget about the intermediate name $i_{0}$, and denote, from now on, the latter solution by $\widetilde{\Delta^{-1}} \bar{\tau} \equiv i_{0}$, or, in more explicit terms,

$$
\widetilde{\Delta^{-1}} \bar{\tau}(\mathbf{x}, t)=\underset{B=0}{\mathrm{FP}} \Delta^{-1}\left[|\widetilde{\mathbf{x}}|^{B} \bar{\tau}(\mathbf{x}, t)\right]
$$

where $\Delta^{-1}$ refers to the standard Poisson integral, and the finite-part symbol $\underset{B=0}{\mathrm{FP}}$ means the previous operations of considering the Laurent expansion when $B \rightarrow 0$, and picking up the finite-part coefficient. Thus, we have proved that $\Delta\left[\widetilde{\Delta^{-1}} \bar{\tau}\right]=\bar{\tau}$, so the generalized inverse Poisson operator $\widetilde{\Delta^{-1}}$ defines a particular solution of the Poisson equation, which has, by construction, none of the problems of divergencies of Poisson integrals which have so much plagued the standard post-Newtonian approximation 819$]$.

Finally let us prove that our generalized solution $\widetilde{\Delta^{-1}} \bar{\tau}$ owns the same properties (B1)(B2) as the corresponding source $\bar{\tau}$. This verification is important because it will allow us to iterate any number of times the operator $\widetilde{\Delta^{-1}}$, and to obtain the post-Newtonian expansion up to any post-Newtonian order. The main problem amounts to prove that $\widetilde{\Delta^{-1}} \bar{\tau}$ admits the same type of expansion at infinity $|\mathbf{x}| \rightarrow+\infty$ as in Eq. (B2). To do this we consider again the same split into near-zone and far-zone contributions : $\widetilde{\Delta^{-1}} \bar{\tau}=I_{<}+I_{>}$, where 


$$
\begin{aligned}
& I_{<}(\mathbf{x}, t)=-\frac{1}{4 \pi} \underset{B=0}{\mathrm{FP}} \int_{|\mathbf{y}|<\mathcal{R}} \frac{d^{3} \mathbf{y}}{|\mathbf{x}-\mathbf{y}|}|\widetilde{\mathbf{y}}|^{B} \bar{\tau}(\mathbf{y}, t), \\
& I_{>}(\mathbf{x}, t)=-\frac{1}{4 \pi} \mathrm{FP}_{B=0} \int_{|\mathbf{y}|>\mathcal{R}} \frac{d^{3} \mathbf{y}}{|\mathbf{x}-\mathbf{y}|}|\widetilde{\mathbf{y}}|^{B} \bar{\tau}(\mathbf{y}, t) .
\end{aligned}
$$

The near-zone integral admits an expansion at infinity which is of the required type. Indeed, because the integrand is of compact support, $|\mathbf{y}|<\mathcal{R}$, we can replace in it the factor $\frac{1}{|\mathbf{x}-\mathbf{y}|}$ by its expansion $\sum \frac{(-)^{l}}{l !} y^{L} \hat{\partial}_{L} \frac{1}{|\mathbf{x}|}$ and integrate term by term. So we have, $\forall N \in \mathbb{N}$,

$$
I_{<}(\mathbf{x}, t)=-\frac{1}{4 \pi} \sum_{l=0}^{N-1} \frac{(-)^{l}}{l !} \hat{\partial}_{L}\left(\frac{1}{r}\right) \underset{B=0}{\mathrm{FP}} \int_{|\mathbf{y}|<\mathcal{R}} d^{3} \mathbf{y}|\widetilde{\mathbf{y}}|^{B} y^{L} \bar{\tau}(\mathbf{y}, t)+\mathcal{O}\left(\frac{1}{r^{N}}\right) .
$$

The right-hand-side has indeed the same structure as in Eq. (B2). The treatment of the far-zone integral is more delicate. We proceed in a way similar to what was done in Eqs. (B5)-(B9). Namely we replace into it the source $\bar{\tau}$ by its expansion given by Eq. (B2). This yields (the finite part of) Eq. (B5), that for convenience we reproduce here :

$$
I_{>}(\mathbf{x}, t)=-\frac{1}{4 \pi} \underset{B=0}{\mathrm{FP}} \int_{|\mathbf{y}|>\mathcal{R}} \frac{d^{3} \mathbf{y}|\widetilde{\mathbf{y}}|^{B}}{|\mathbf{x}-\mathbf{y}|}\left\{\sum \hat{n}_{L}(\mathbf{y})|\mathbf{y}|^{a}(\ln |\mathbf{y}|)^{p} G_{L, a, p}(t)+S_{N}(\mathbf{y}, t)\right\} .
$$

There is a contribution of the remainder and a finite sum of terms with known structure. The remainder contribution is simply given by the value at $B=0$ which has been written in the right-hand-side of Eq. (B6). Let us write this term in the form

$$
\int_{|\mathbf{y}|>\mathcal{R}} \frac{d^{3} \mathbf{y}}{|\mathbf{x}-\mathbf{y}|} S_{N}(\mathbf{y}, t)=\sum_{l=0}^{N-4} \frac{(-)^{l}}{l !} \hat{\partial}_{L}\left(\frac{1}{r}\right) \int_{|\mathbf{y}|>\mathcal{R}} d^{3} \mathbf{y} y^{L} S_{N}(\mathbf{y}, t)+T_{N-2}(\mathbf{x}, t)
$$

where we introduced the $N-4$ first terms of the multipolar expansion of $\frac{1}{|\mathbf{x}-\mathbf{y}|}$ when $r=$ $|\mathbf{x}| \rightarrow+\infty$, and where

$$
T_{N-2}(\mathbf{x}, t)=\int_{|\mathbf{y}|>\mathcal{R}} d^{3} \mathbf{y}\left[\frac{1}{|\mathbf{x}-\mathbf{y}|}-\sum_{l=0}^{N-4} \frac{(-)^{l}}{l !} y^{L} \hat{\partial}_{L}\left(\frac{1}{r}\right)\right] S_{N}(\mathbf{y}, t) .
$$

The maximal order $N-4$ of the expansion is chosen in such a way that all the terms in Eq. (B23) are given by convergent integrals at infinity, owing to the fact that the remainder 
satisfies $S_{N}=\mathcal{O}\left(1 / r^{N}\right)$. Now we prove that $T_{N-2}$, defined by Eq. (B23), is also a remainder in the sense that $T_{N-2}=\mathcal{O}\left(\ln r / r^{N-2}\right)$. We split $T_{N-2}$ into two integrals, a near-zone integral $T_{N-2}^{\text {near }}$ corresponding to the integration range $\left.|\mathbf{y}| \in\right] \mathcal{R},|\mathbf{x}|\left[\right.$, and a far-zone one $T_{N-2}^{\mathrm{far}}$ corresponding to $|\mathbf{y}| \in]|\mathbf{x}|,+\infty[$. In the near-zone integral we can use the bound

$$
\left|\frac{1}{|\mathbf{x}-\mathbf{y}|}-\sum_{l=0}^{N-4} \frac{(-)^{l}}{l !} y^{L} \hat{\partial}_{L}\left(\frac{1}{r}\right)\right| \leq C_{N} \frac{|\mathbf{y}|^{N-3}}{|\mathbf{x}|^{N-2}}
$$

where $C_{N}$ is a constant. On the other hand, because $S_{N}=\mathcal{O}\left(1 / r^{N}\right)$, there is also a constant $A_{N}$, depending on the value of $\mathcal{R}$, such that the following majoration holds :

$$
\left|S_{N}(\mathbf{y}, t)\right| \leq \frac{A_{N}}{|\mathbf{y}|^{N}}
$$

Replacing these results into the near-zone integral we get

$$
\left|T_{N-2}^{\text {near }}(\mathbf{x}, t)\right| \leq 4 \pi \frac{A_{N} C_{N}}{|\mathbf{x}|^{N-2}} \ln \left(\frac{|\mathbf{x}|}{\mathcal{R}}\right)
$$

In the far-zone integral, we can no longer apply the bound (B24) but still we can employ the majoration (B25). Then we can easily show the inequality (in which $|\mathbf{y}|=|\mathbf{x}| \lambda$ )

$$
\left|T_{N-2}^{\mathrm{far}}(\mathbf{x}, t)\right| \leq 4 \pi \frac{A_{N}}{|\mathbf{x}|^{N-2}} \int_{1}^{+\infty} \frac{d \lambda}{\lambda^{N-2}}\left[\frac{1}{\lambda}+\sum_{l=0}^{N-4} \frac{(2 l-1) ! !}{l !} \lambda^{l}\right] .
$$

The integral is convergent. At last, from Eqs. (B26) and (B27) we have proved that $T_{N-2}=\mathcal{O}\left(\ln r / r^{N-2}\right)$. Still it remains to show that the finite sum of terms in Eq. (B21), i.e. besides the remainder, admits some expansions of the required structure. But this follows from applying the finite part operation $\underset{B=0}{\mathrm{FP}}$ onto the result ( $\overline{\mathrm{B} 9}$ ), which tells us immediately that we have an expansion of the correct type $\sim \hat{n}_{L}(\mathbf{x})|\mathbf{x}|^{a}(\ln |\mathbf{x}|)^{q}$. 


\section{APPENDIX C: FAR ZONE EXPANSION OF THE POISSON INTEGRAL}

Thanks to the investigation in Appendix B, the far-zone (or multipolar) expansion of the object $\widetilde{\Delta^{-1}}[\bar{\tau}]$ happens to be workable. Recall that controlling the far-zone expansion of the post-Newtonian field is fundamental since it is at the basis of the matching. The operation of taking the far-zone expansion is denoted $\mathcal{M}$ when applied on post-Newtonian objects (see Section $\llbracket$ IIB $)$. We therefore want to determine the expression of $\mathcal{M}\left(\widetilde{\Delta^{-1}}[\bar{\tau}]\right)$. That is, we want to relate it to the expansion of the corresponding source, which has the same structure as in Eq. (3.22) :

$$
\mathcal{M}(\bar{\tau})(\mathbf{x}, t)=\sum \hat{n}_{L} r^{a}(\ln r)^{p} G_{L, a, p}(t)
$$

By the matching equation we know that this far-zone expansion is identical with the nearzone expansion of the external field [see e.g. Eq. (A3)]. Let us first apply $\mathcal{M}$ onto $\widetilde{\Delta^{-1}}[\bar{\tau}]$ as expressed as a sum of near-zone and far-zone contributions,

$$
\mathcal{M}\left(\widetilde{\Delta^{-1}}[\bar{\tau}]\right)=\mathcal{M}\left(I_{<}\right)+\mathcal{M}\left(I_{>}\right)
$$

where $I_{<}$and $I_{>}$are defined by Eqs. (B18)-(B19). The near-zone integral is quite easy to work with. Indeed, from Eq. (B20) we see that its expansion when $r=|\mathbf{x}| \rightarrow+\infty$ is obtained by expanding the factor $1 /|\mathbf{x}-\mathbf{y}|$ inside the integrand. Therefore, the infinite far-zone expansion (without remainder) reads

$$
\mathcal{M}\left(I_{<}\right)=-\frac{1}{4 \pi} \underset{B=0}{\mathrm{FP}} \int_{|\mathbf{y}|<\mathcal{R}} d^{3} \mathbf{y}|\widetilde{\mathbf{y}}|^{B} \mathcal{M}\left(\frac{1}{|\mathbf{x}-\mathbf{y}|}\right) \bar{\tau}(\mathbf{y}, t)
$$

in which we denote

$$
\mathcal{M}\left(\frac{1}{|\mathbf{x}-\mathbf{y}|}\right)=\sum_{l=0}^{+\infty} \frac{(-)^{l}}{l !} y_{L} \hat{\partial}_{L}\left(\frac{1}{|\mathbf{x}|}\right)
$$


On the other hand, the far-zone expansion of the far-zone integral $I_{>}$has been obtained in Eq. (B21)-(B22), where we found that it comes from replacing the source term by its far-zone expansion [indeed, when $\mathcal{R}$ is large enough, the integration ranges over the domain of validity of the far-zone expansion]. So the infinite far-zone expansion of that term is given by

$$
\mathcal{M}\left(I_{>}\right)=-\frac{1}{4 \pi} \underset{B=0}{\mathrm{FP}} \int_{|\mathbf{y}|>\mathcal{R}} \frac{d^{3} \mathbf{y}|\widetilde{\mathbf{y}}|^{B}}{|\mathbf{x}-\mathbf{y}|} \mathcal{M}(\bar{\tau}(\mathbf{y}, t)),
$$

where the integrand contains the expansion of the source given by Eq. (C1). Now let us use a technical lemma which is quite important in the present formalism, and has already played a crucial role in Refs. [39,40]. This lemma is based on the remark that any radial integral of the type $\int_{0}^{+\infty} d|\mathbf{y}||\mathbf{y}|^{B+a}(\ln |\mathbf{y}|)^{p}$, where $B \in \mathbb{C}$ and $a$ and $p$ are arbitrary real numbers, is identically zero by analytic continuation in B. See Ref. 51 for the proof. Our useful lemma, that is trivial to relate to the previous remark (after performing the integration over angles), is

$$
\underset{B=0}{\mathrm{FP}} \int d^{3} \mathbf{y}|\widetilde{\mathbf{y}}|^{B} \mathcal{M}\left(\frac{1}{|\mathbf{x}-\mathbf{y}|}\right) \mathcal{M}(\bar{\tau}(\mathbf{y}, t))=0
$$

The point here is that the integral ranges over the complete three-dimensional space $\mathbb{R}^{3}$. Now we have the "numerical" equalities $\mathcal{M}\left(\frac{1}{|\mathbf{x}-\mathbf{y}|}\right)=\frac{1}{|\mathbf{x}-\mathbf{y}|}$ when $|\mathbf{y}|<|\mathbf{x}|$ and $\mathcal{M}(\bar{\tau})=\bar{\tau}$ when $|\mathbf{y}|>a$, where $a$ is the radius of the compact-support source. From this we deduce that as soon as $\mathcal{R}>a$, what we can always assume right from the beginning, and $|\mathbf{x}|>\mathcal{R}$, which is not a problem because we are considering the limit $|\mathbf{x}| \rightarrow+\infty$, we have the identity

$$
\underset{B=0}{\mathrm{FP}} \int_{|\mathbf{y}|<\mathcal{R}} \frac{d^{3} \mathbf{y}|\widetilde{\mathbf{y}}|^{B}}{|\mathbf{x}-\mathbf{y}|} \mathcal{M}(\bar{\tau}(\mathbf{y}, t))+\underset{B=0}{\mathrm{FP}} \int_{|\mathbf{y}|>\mathcal{R}} d^{3} \mathbf{y}|\widetilde{\mathbf{y}}|^{B} \mathcal{M}\left(\frac{1}{|\mathbf{x}-\mathbf{y}|}\right) \bar{\tau}(\mathbf{y}, t)=0 .
$$

By means of that identity we can obtain the requested form of the far-zone expansion as

$$
\mathcal{M}\left(\widetilde{\Delta^{-1}}[\bar{\tau}]\right)=-\frac{1}{4 \pi} \underset{B=0}{\mathrm{FP}} \int d^{3} \mathbf{y}|\widetilde{\mathbf{y}}|^{B}\left[\frac{1}{|\mathbf{x}-\mathbf{y}|} \mathcal{M}(\bar{\tau}(\mathbf{y}, t))+\mathcal{M}\left(\frac{1}{|\mathbf{x}-\mathbf{y}|}\right) \bar{\tau}(\mathbf{y}, t)\right]
$$


In this particular form we see that the $\mathcal{M}$-operator is distributed on the two terms like a derivative operator would be. In the first term we recognize the action of the generalized Poisson integral. Actually this Poisson operator has been defined in Appendix A when acting on a near-zone expansion of the type (A3), but by matching that expansion is the same as the present far-zone expansion, so the definition is rigorously the same. Finally we can re-write Eq. (C8) into the alternative form

$$
\mathcal{M}\left(\widetilde{\Delta^{-1}}[\bar{\tau}]\right)=\widetilde{\Delta^{-1}}[\mathcal{M}(\bar{\tau})]-\frac{1}{4 \pi} \sum_{l=0}^{+\infty} \frac{(-)^{l}}{l !} \hat{\partial}_{L}\left(r^{-1}\right) \underset{B=0}{\mathrm{FP}} \int d^{3} \mathbf{y} \mid \widetilde{\mathbf{y}}^{B} \hat{y}_{L} \bar{\tau}(\mathbf{y}, t)
$$

which constitutes the main result of this Appendix. Notice that Eq. (C9) is in agreement with the multipole expansion of the retarded integral as given by Eq. (3.11)-(3.12) in Ref. [40], when specialized to the static case where there is no dependence on time.

Next we derive the analogous result concerning the operator of the "instantaneous" potentials

$$
\widetilde{\mathcal{I}^{-1}}=\sum_{k=0}^{+\infty} \frac{1}{c^{2 k}} \partial_{t}^{2 k} \widetilde{\Delta^{-k-1}}
$$

We iterate $k+1$ times the result (C9). There is no problem for doing this; the only point is that we use in a repeated way the easily checked formula telling that we are allowed to "operate by parts" the Poisson integral $\widetilde{\Delta^{-1}}$ in the way

$$
\underset{B=0}{\mathrm{FP}} \int d^{3} \mathbf{z}|\widetilde{\mathbf{z}}|^{B} \hat{z}_{L} \widetilde{\Delta^{-1}} \bar{\tau}=\underset{B=0}{\mathrm{FP}} \int d^{3} \mathbf{y}|\widetilde{\mathbf{y}}|^{B} \widetilde{\Delta^{-1}}\left[\hat{y}_{L}\right] \bar{\tau}
$$

This formula is a consequence of the fact that $\underset{B=0}{\mathrm{FP}} \int d^{3} \mathbf{z}|\widetilde{\mathbf{z}}|^{B} \frac{\hat{z}_{L}}{|\mathbf{z}-\mathbf{y}|}=-4 \pi \widetilde{\Delta^{-1}}\left[\hat{y}_{L}\right]=$ $\frac{-2 \pi}{2 l+3}|\mathbf{y}|^{2} \hat{y}_{L} ;$ see Eq. (4.10) in Ref. [43]. Therefore we arrive at

$$
\begin{aligned}
\mathcal{M}\left(\widetilde{\Delta^{-k-1}}[\bar{\tau}]\right) & =\widetilde{\Delta^{-k-1}}[\mathcal{M}(\bar{\tau})] \\
& -\frac{1}{4 \pi} \sum_{l=0}^{+\infty} \frac{(-)^{l}}{l !} \sum_{i=0}^{k} \widetilde{\Delta^{-i}}\left[\hat{\partial}_{L}\left(r^{-1}\right)\right] \underset{B=0}{\mathrm{FP}} \int d^{3} \mathbf{y}|\widetilde{\mathbf{y}}|^{B} \widetilde{\Delta^{i-k}}\left[\hat{y}_{L}\right] \bar{\tau}(\mathbf{y}, t)
\end{aligned}
$$


and from this it is very simple to derive the requested expression concerning $\widetilde{\mathcal{I}^{-1}}$. We obtain

$$
\begin{aligned}
\mathcal{M}\left(\widetilde{\mathcal{I}^{-1}}[\bar{\tau}]\right) & =\widetilde{\mathcal{I}^{-1}}[\mathcal{M}(\bar{\tau})] \\
& -\frac{1}{4 \pi} \sum_{l=0}^{+\infty} \frac{(-)^{l}}{l !} \sum_{i=0}^{+\infty} \widetilde{\Delta^{-i}}\left[\hat{\partial}_{L}\left(r^{-1}\right)\right] \sum_{k=i}^{+\infty} \frac{1}{c^{2 k}} \mathrm{FP}_{B=0} \int d^{3} \mathbf{y}|\widetilde{\mathbf{y}}|^{B} \widetilde{\Delta^{i-k}}\left[\hat{y}_{L}\right] \partial_{t}^{2 k} \bar{\tau}(\mathbf{y}, t)
\end{aligned}
$$

This expression, though completely explicit, does not constitute our final form. Because the "instantaneous" solution is a particular solution of the d'Alembertian equation, it must be possible to re-express the second term in Eq. (C13) as a combination of some source-free retarded and advanced multipolar waves. To see this we notice that

$$
\widetilde{\Delta^{-i}}\left[\hat{\partial}_{L}\left(r^{-1}\right)\right]=\hat{\partial}_{L}\left(\frac{r^{2 i-1}}{(2 i) !}\right)
$$

which shows that the latter homogeneous solution is actually one of the symmetric type, i.e. retarded plus advanced. Namely we can re-write Eq. (C13) into the form

$$
\mathcal{M}\left(\widetilde{\mathcal{I}^{-1}}[\bar{\tau}]\right)=\widetilde{\mathcal{I}^{-1}}[\mathcal{M}(\bar{\tau})]-\frac{1}{4 \pi} \sum_{l=0}^{+\infty} \frac{(-)^{l}}{l !} \hat{\partial}_{L}\left\{\frac{\overline{\mathcal{F}_{L}(t-r / c)+\mathcal{F}_{L}(t+r / c)}}{2 r}\right\}
$$

where the overline notation means taking the Taylor expansion of the symmetric wave when the retardation $r / c \rightarrow 0$ [the result is displayed in Eq. (3.24)]. Actually, this overline notation is somewhat misleading, because, in keeping with the real meaning of the result (C15), one should a posteriori interpret the latter Taylor expansion as a far-zone (singular) expansion when $r \rightarrow+\infty$. However, in view of the matching, it is more fruitful to employ the same overline notation as for the expansion of the anti-symmetric waves occuring in the near-zone metric - indeed the matching is simply interested at identifying together some asymptotic expansions which are of the same form. The "multipole-moment" function $\mathcal{F}_{L}(t)$ in Eq. (C15) is given by

$$
\mathcal{F}_{L}(t)=\sum_{j=0}^{+\infty} \frac{1}{c^{2 j}} \underset{B=0}{\mathrm{FP}} \int d^{3} \mathbf{y}|\widetilde{\mathbf{y}}|^{B} \widetilde{\Delta^{-j}}\left[\hat{y}_{L}\right] \partial_{t}^{2 j} \bar{\tau}(\mathbf{y}, t)
$$


Finally let us find an alternative, more compact, form for this result. We introduce the l-dependent function

$$
\delta_{l}(z)=\frac{(2 l+1) ! !}{2^{l+1} l !}\left(1-z^{2}\right)^{l}
$$

whose integral is normalized to one : $\int_{-1}^{1} d z \delta_{l}(z)=1$. One can readily show that

$$
\widetilde{\Delta^{-j}}\left[\hat{y}_{L}\right]=|\mathbf{y}|^{2 j} \hat{y}_{L} \int_{-1}^{1} d z \frac{z^{2 j}}{(2 j) !} \delta_{l}(z)
$$

which permits to express the function $\mathcal{F}_{L}$ in a form where the post-Newtonian series is formally re-summed as

$$
\mathcal{F}_{L}(t)=\underset{B=0}{\mathrm{FP}} \int d^{3} \mathbf{x}|\widetilde{\mathbf{y}}|^{B} \hat{y}_{L} \overline{\int_{-1}^{1} d z \delta_{l}(z) \bar{\tau}(\mathbf{y}, t \pm z|\mathbf{y}| / c)}
$$

Under this form we recognize the multipole-moment function introduced in Eq. (3.14) in Ref. [40] (the function remains unchanged by taking either sign \pm in the time argument of $\bar{\tau})$. This result permits to fully determine the exterior multipolar field by matching, and to recover the expression already obtained in Ref. 40 by means of a somewhat different method. 


\section{REFERENCES}

[1] A. Einstein, Sitzber. Preuss. Akad. Wiss. (Berlin), 688 (1916).

[2] J.Droste,Versl. K. Akad. Wet. Amsterdam 25, 460 (1916).

[3] W. DeSitter, Mon. Not. R. A. S. 76, 699 and 77, 155 (1916).

[4] A. Einstein, L. Infeld and B. Hoffmann, Ann. Math. 39, 65 (1938).

[5] H.A. Lorentz and J.Droste, Versl. K. Akad. Wet. Amsterdam 26, 392 and 649 (1917); in the collected papers of H.A. Lorentz, vol. 5, The Hague, Nijhoff (1937).

[6] V. Fock, J. Phys. (U.S.S.R.) 1, 81 (1939).

[7] V.A. Fock, Theory of Space, Time and Gravitation, Pergamon, London (1959).

[8] S. Chandrasekhar, Astrophys. J. 142, 1488 (1965).

[9] S. Chandrasekhar and Y. Nutku, Astrophys. J. 158, 55 (1969).

[10] S. Chandrasekhar and F.P. Esposito, Astrophys. J. 160, 153 (1970).

[11] J.L. Anderson and T.C. DeCanio, Gen. Relat. Grav. 6, 197 (1975).

[12] J. Ehlers, in the Proc. of the International school of Relativistic Astrophysics, J. Ehlers (ed.), Erice, MPI:München (1977).

[13] J. Ehlers, Ann. N.Y. Acad. Sci. 336, 279 (1980).

[14] G.D. Kerlick, Gen. Rel. Grav. 12, 467 (1980).

[15] G.D. Kerlick, Gen. Rel. Grav. 12, 521 (1980).

[16] A. Caporali, Nuovo Cimento 61B, 181 (1981).

[17] A. Papapetrou and B. Linet, Gen. Relat. Grav. 13, 335 (1981).

[18] R. Breuer and E. Rudolph, Gen. Rel. Grav. 13, 777 (1981). 
[19] R. Breuer and E. Rudolph, Gen. Rel. Grav. 14, 181 (1982).

[20] T. Damour and N. Deruelle, Phys. Lett. 87A, 81 (1981).

[21] T. Damour, C. R. Acad. Sc. Paris 294, 1355 (1982).

[22] T. Damour, in Gravitational Radiation, N. Deruelle and T. Piran (eds.), North-Holland Company (1983).

[23] L. Blanchet, G. Faye and B. Ponsot, Phys. Rev. D58, 124002 (1998).

[24] P. Jaranowski and G. Schäfer, Phys. Rev. D57, 7274 (1998).

[25] T. Damour, P. Jaranowski and G. Schäfer, Phys. Rev. D62, 021501R (2000); Erratum Phys. Rev. D63, 029903 (2001).

[26] L. Blanchet and G. Faye, Phys. Lett. A 271, 58 (2000).

[27] L. Blanchet and G. Faye, Phys. Rev. D63, 062005 (2001).

[28] V. C. de Andrade, L. Blanchet and G. Faye, Class. Quantum Grav. 18, 753 (2001).

[29] W.L. Burke and K.S. Thorne, in Relativity, M. Carmeli et al. (eds), Plenum Press, New York, p. 208-209 (1970).

[30] W.L. Burke, J. Math. Phys. 12, 401 (1971).

[31] L. Blanchet and T. Damour, Phys. Rev. D37, 1411 (1988).

[32] L. Blanchet, Phys. Rev. D47, 4392 (1993).

[33] L. Blanchet, Phys. Rev. D55, 714 (1997).

[34] B.R. Iyer and C.M. Will, Phys. Rev. Lett. 70, 113 (1993).

[35] B.R. Iyer and C.M. Will, Phys. Rev. D52, 6882 (1995).

[36] L. Blanchet and T. Damour, Philos. Trans. R. Soc. London A320, 379 (1986). 
[37] L. Blanchet and T. Damour, Ann. Inst. Henri Poincaré A 50, 377 (1989).

[38] T. Damour and B.R. Iyer, Ann. Inst. Henri Poincaré A 54, 115 (1991).

[39] L. Blanchet, Phys. Rev. D 51, 2559 (1995).

[40] L. Blanchet, Class. Quantum. Grav. 15, 1971 (1998).

[41] L. Blanchet, T. Damour and B.R. Iyer, Phys. Rev. D 51, 5360 (1995).

[42] C.M. Will and A.G. Wiseman, Phys. Rev. D 54, 4813 (1996).

[43] L. Blanchet, Phys. Rev. D 54, 1417 (1996).

[44] L. Blanchet, Class. Quantum. Grav. 15, 113 (1998).

[45] L. Blanchet, B.R. Iyer and B. Joguet, Phys. Rev. D 65, 064005 (2002).

[46] A.D. Rendall, Proc. R. Soc. London A438, 341 (1992).

[47] T. Futamase and B. F. Schutz, Phys. Rev. D 28, 2363 (1983).

[48] T. Futamase, Phys. Rev. D 28, 2373 (1983).

[49] Our notation for STF tensors is the following. $L=i_{1} i_{2} \cdots i_{l}$ denotes a multi-index, made of $l$ (spatial) indices. When summing over multi-indices we never write the $l$ summations over the $l$ indices $i_{1}, \cdots, i_{l}$ ranging from 1 to 3 . The STF product of unit vectors $n_{i}=n^{i} \equiv x^{i} / r$ is denoted $\hat{n}_{L}=\operatorname{STF}\left(n_{L}\right)$, where $n_{L}$ is a short-hand for $n_{i_{1}} \cdots n_{i_{l}}$. For instance $\hat{n}_{i j}=n_{i} n_{j}-\frac{1}{3} \delta_{i j}$. Similarly we denote $x_{L}=x_{i_{1}} \cdots x_{i_{l}}=r^{l} n_{L}$ and $\hat{x}_{L}=\operatorname{STF}\left(x_{L}\right)$. The derivative operator $\partial_{L}$ is a short-hand for $\partial_{i_{1}} \cdots \partial_{i_{l}}$, and we have $\hat{\partial}_{L}=\operatorname{STF}\left(\partial_{L}\right)$. For instance $\hat{\partial}_{i j}=\partial_{i j}-\frac{1}{3} \delta_{i j} \Delta$. More generally, a function $F_{L}$ is said to be STF with respect to the $l$ indices composing $L$ if and only if, for any pair of indices $i_{p}, i_{q} \in L$, we have $F_{\ldots i_{p} \cdots i_{q} \cdots}=F_{\cdots i_{q} \cdots i_{p} \cdots}$ and $\delta_{i_{p} i_{q}} F_{\ldots i_{p} \cdots i_{q} \cdots}=0$ (see the Appendices A and B in Ref. [36] for reviews about the STF formalism).

[50] It is clear that for stationary sources (independent of time), the anti-symmetric waves 
given by Eqs. (2.22) are zero. Therefore the only contribution to the function $\mathcal{R}_{L}^{\mu \nu}(t)$ comes from the non-stationary (or radiative) part of the field, which according to our assumption of stationarity in the past is zero when $t \leq-\mathcal{T}$, and for which $\mathcal{R}_{L}^{\mu \nu}(t)$ is perfectly well-defined. For simplicity in the notation we do not indicate that $\mathcal{R}_{L}^{\mu \nu}(t)$ should be computed only from the "radiative" part of the source term $\mathcal{M}\left(\tau^{\mu \nu}\right)$.

[51] We want to prove that the radial integral $\int_{0}^{+\infty} d|\mathbf{y}||\mathbf{y}|^{B+a}(\ln |\mathbf{y}|)^{p}$ is zero by analytic continuation $(\forall B \in \mathbb{C})$. First we can get rid of the logarithms by considering some repeated differentiations with respect to $B$; thus we need only to consider the simpler integral $\int_{0}^{+\infty} d|\mathbf{y}||\mathbf{y}|^{B+a}$. We split the integral into a near-zone integral $\int_{0}^{\mathcal{R}} d|\mathbf{y}||\mathbf{y}|^{B+a}$ and a far-zone one $\int_{\mathcal{R}}^{+\infty} d|\mathbf{y}||\mathbf{y}|^{B+a}$, where $\mathcal{R}$ is some constant radius. When $\Re(B)$ is a large enough positive number, the value of the near-zone integral is $\mathcal{R}^{B+a+1} /(B+a+1)$, while when $\Re(B)$ is a large negative number, the far-zone integral reads the opposite, $-\mathcal{R}^{B+a+1} /(B+a+1)$. Both obtained values represent the unique analytic continuations of the near-zone and far-zone integrals for any $B \in \mathbb{C}$ except $-a-1$. The complete integral $\int_{0}^{+\infty} d|\mathbf{y}||\mathbf{y}|^{B+b}$ is equal to the sum of these analytic continuations, and is therefore identically zero $(\forall B \in \mathbb{C}$, including the value $-a-1)$. 